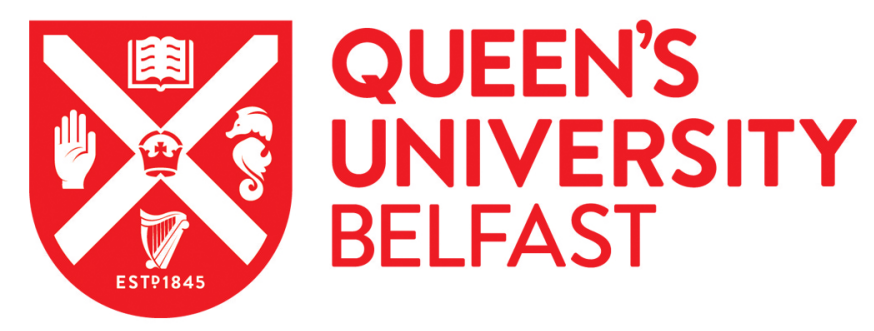

\title{
Massive MIMO Asymptotics for Ray-Based Propagation Channels
}

Li, S., Smith, P., Dmochowski , P. A., Tataria, H., Matthaiou, M., \& Yin, J. (2020). Massive MIMO Asymptotics for Ray-Based Propagation Channels. IEEE Transactions on Wireless Communications.

https://doi.org/10.1109/TWC.2020.2979146

\section{Published in:}

IEEE Transactions on Wireless Communications

\section{Document Version:}

Peer reviewed version

\section{Queen's University Belfast - Research Portal:}

Link to publication record in Queen's University Belfast Research Portal

\section{Publisher rights}

(c) 2020 IEEE.

This work is made available online in accordance with the publisher's policies. Please refer to any applicable terms of use of the publisher.

\section{General rights}

Copyright for the publications made accessible via the Queen's University Belfast Research Portal is retained by the author(s) and / or other copyright owners and it is a condition of accessing these publications that users recognise and abide by the legal requirements associated with these rights.

Take down policy

The Research Portal is Queen's institutional repository that provides access to Queen's research output. Every effort has been made to ensure that content in the Research Portal does not infringe any person's rights, or applicable UK laws. If you discover content in the Research Portal that you believe breaches copyright or violates any law, please contact openaccess@qub.ac.uk. 


\title{
Massive MIMO Asymptotics for Ray-Based Propagation Channels
}

\author{
Shuang Li, Student Member, IEEE, Peter Smith, Fellow, IEEE, Pawel Dmochowski, Senior Member, IEEE, Harsh \\ Tataria, Member, IEEE, Michail Matthaiou, Senior Member, IEEE, and Jingwei Yin, Member, IEEE
}

\begin{abstract}
Favorable propagation (FP) and channel hardening (CH) are desired properties in massive multiple-input multipleoutput (MIMO) systems. To date, these properties have primarily been analyzed for classical statistical channel models, or ray-based models with very specific angular parameters and distributions. This paper presents a thorough mathematical analysis of the asymptotic system behavior for ray-based channels with arbitrary ray distributions, and considers two types of antenna array structures at the cellular base station: a uniform linear array (ULA) and a uniform planar array (UPA). In addition to FP and channel hardening, we analyze the large system potential (LSP) which measures the asymptotic ratio of the expected power in the desired channel to the expected total interference power when both the antenna and user numbers grow. LSP is said to hold when this ratio converges to a positive constant. The results demonstrate that while FP is guaranteed in ray-based channels, CH may or may not occur depending on the nature of the model. Furthermore, we demonstrate that LSP will not normally hold as the expected interference power grows logarithmically for both ULAs and UPAs relative to the power in the desired channel as the system size increases. Nevertheless, we identify some fundamental and attractive properties of massive MIMO in this limiting regime.
\end{abstract}

Index Terms-Massive MIMO, Favourable Propagation, Channel Hardening, Ray-based, ULA, UPA.

\section{INTRODUCTION}

Two key principles behind the success of massive MIMO are favorable propagation (FP) [2], [3], and channel hardening $(\mathrm{CH})$ [4], meaning that the normalized inter-user interference power converges to zero, and that the normalized power in the desired channel becomes constant. With FP, the use of large numbers of antennas offers an implicit interference reduction mechanism, and enables the use of low complexity signal processing algorithms [3], [5], [6].

Parts of this work were presented at the IEEE International Conference on Communications (ICC) 2019 [1].

The work of S. Li was supported by China Scholarship Council. The work of M. Matthaiou was supported by EPSRC, UK, under grant EP/P000673/1.

$\mathrm{S}$. Li and P. A. Dmochowski are with the School of Engineering and Computer Science, Victoria University of Wellington, PO Box 600 Wellington 6140, New Zealand (e-mail: \{lishua, pawel.dmochowski\}@ecs.vuw.ac.nz).

P. J. Smith is with the School of Mathematics and Statistics, Victoria University of Wellington, PO Box 600 Wellington 6140, New Zealand (email: peter.smith@ecs.vuw.ac.nz).

H. Tataria is with the Department of Electrical and Information Technology, Lund University, Lund, Sweden (e-mail: harsh.tataria@eit.ith.se).

M. Matthaiou is with the Institute of Electronics, Communications and Information Technology (ECIT), Queen's University Belfast, Belfast, U.K. (e-mail: m.matthaiou@qub.ac.uk).

J. Yin is with the College of Underwater Acoustic Engineering, Harbin Engineering University, Harbin 150001, P.R.China (e-mail: yinjingwei@hrbeu.edu.cn).
The bulk of the theoretical work on FP and $\mathrm{CH}$ has utilized classical statistical channel models. Here, the existence of FP has been demonstrated for channel models of increasing complexity, progressing from independent and identically distributed (i.i.d.) Rayleigh [2], [7], pure line-of-sight [2], [7], correlated Rayleigh [8], [9], and independent Ricean [10] to correlated Ricean channels [11], [12]. In parallel, with the theory, channel measurements have demonstrated that a large fraction of the theoretical gains due to FP can be obtained [13]-[15].

This work is now mature, but incomplete in the sense that accurate modeling of large dimensional channels requires a strong link to the propagation environment. This is usually obtained through ray-based models which have been extensively validated by measurements and, for this reason, have been adapted in the 3GPP standardization [16]. These models better capture the physics of electromagnetic propagation, have a closer link to the array architecture and are widely used irrespective of the frequency band [16]-[18]. The physical nature of the ray-based models also has advantages in system performance analysis since the analytical conclusions are based on physical features of the system rather than statistical modeling assumptions. For example, FP was considered in the recent work [11] for very general heterogeneous, correlated Ricean channels. This work gives wide ranging FP results, but the inherent nature of these models meant that the conclusions relied on various assumptions concerning the correlation structure, line-of-sight direction, etc. In contrast, we are able to prove FP for ray-based models with the most basic assumptions pertaining to physical phenomena, such as ray direction distributions.

Variations of such models have a proliferation of names including directional, spatial and Saleh-Valenzulela (SV) type [19] channel models. We prefer the phrase ray-based, as the main requirement for our work is that the statistical distributions of individual rays can be identified and analyzed. ${ }^{1}$ This is possible for a wide range of such channels. Important work has begun in this area demonstrating the existence of FP with specific ray-based models for a variety of antenna topologies, such as the uniform linear array (ULA), uniform planar array (UPA), and uniform circular array (UCA) [20], [21]. However, the majority of this work relies on two very special cases for the rays: an arbitrary ray must arrive with an azimuth angle, $\phi$, which satisfies $\phi \sim U[0,2 \pi]$ [20], [22]

\footnotetext{
${ }^{1}$ We prefer this terminology, since our primary interest is not in identifying distributions of rays across multiple clusters.
} 
or $\sin \phi \sim U[-1,1]$ [23]. FP has also been demonstrated in [24] for the more complex case where azimuth angles have a uniform central angle and wrapped Gaussian sub rays and the elevation angles are Laplacian. However, with the exception of our work in [1], there are no general results available for arbitrary angular distributions. It is, thus, critical to be able to predict the system performance with practical, and more general distributions, making our extension an important one in the context of the understanding of massive MIMO behavior. Hence, a general analysis of FP for ray-based models with arbitrary ray distributions is almost entirely lacking. Further, while FP is a desirable property for a communication system, it only implies that a finite number of users can be served by increasing the number of antenna elements. We refer to this as single-sided massive MIMO [20]. Ideally, as you grow the number of BS antennas you would also serve more users, leading to a system that becomes large both in users and antennas, i.e., double-sided massive MIMO, a concept defined in [25]. Hence, we define large system potential (LSP) as the property that the fundamental ratio which measures the mean power in the desired channel relative to the total mean interference power converges to a positive constant as both the number of users $(K)$ and the number of antennas $(N)$ grow to infinity, with $N / K \rightarrow \alpha$ as $N \rightarrow \infty$. The analysis of LSP for i.i.d. Rayleigh fading can be found in [6], although not explicitly defined. In our conference paper [1], CH, FP, and LSP analysis for ray-based channel models and a basic ULA antenna structure were discussed. Here, we extend our earlier work in [1] by also considering a UPA structure. This is an important, yet non trivial, extension as the addition of the elevation component in the ray based channel facilitates much greater accuracy in predicting massive MIMO behavior, something which is rare in the literature. Furthermore, the majority of the ongoing deployments of massive MIMO in the C-band utilize UPAs, in order to leverage the full dimensional nature of the channel, and to maximize the beamforming gain of the system via reciprocity-based processing. The mathematical complexity of such an extension is substantial, since our main aim is to obtain detailed insights into the desirable properties of massive MIMO systems with a model which is more closely in line with practice. We make the following contributions for both ULA and UPA topologies:

- We show that $\mathrm{CH}$ may or may not occur depending on the nature of the model.

- We show that FP is guaranteed for all models where the ray angles are continuous random variables (as assumed by all models to date).

- For LSP, we derive remarkably simple expressions which relate the asymptotic interference behavior to system size, antenna spacing the ray distribution. We demonstrate that LSP will not normally hold as the mean interference power grows logarithmically in $N$ relative to the mean power of the desired user channel as the system size increases.

- Analytical results are verified via simulation and using exact closed-form special cases derived for specific angular distributions.
- Despite the lack of LSP, the implications for massive MIMO are excellent. Although the interference eventually dominates the desired channel, the growth is very slow and is further attenuated by practical factors such as the likely propagation environment and the typical array patterns employed. In addition, we prove that trivial scheduling schemes can retain LSP, and thus increase the robustness of massive MIMO performance.

Notation. Boldface lower and upper case symbols denote vectors and matrices. Complex conjugation and Hermitian transpose operations are denoted by $(\cdot)^{*}$ and $(\cdot)^{\mathrm{H}}$, respectively, while $\otimes$ denotes the Kronecker product; $\mathcal{C N}(\mathbf{m}, \mathbf{R})$ denotes the circular symmetric complex Gaussian distribution with mean $\mathbf{m}$ and covariance matrix $\mathbf{R}$, while $U[a, b]$ denotes a uniform distribution on $[a, b] ; \mathbb{E}[\cdot]$ denotes statistical expectation, $\sim$ denotes asymptotic equivalence defined in [26, p. 15], $\stackrel{\text { a.s. }}{\longrightarrow}$ denotes almost sure convergence, and $\mathcal{F}(\cdot)$ denotes the Fourier transform. The functions $J_{0}(\cdot)$ and $I_{0}(\cdot)$ denote the zero order Bessel and modified Bessel functions of the first kind, while $O(\cdot)$ denotes the growth rate of the argument. The Dirichlet kernel is defined as $\mathrm{D}_{n}(x)=\frac{\sin ((n+1 / 2) x)}{2 \pi \sin (x / 2)}$ for some positive integer $n$.

\section{Channel Model and System Metrics}

We consider an uplink massive MIMO system with $N$ co-located antennas at one base station (BS) simultaneously serving $K$ single antenna users, where, for now, $N \gg K$. We assume a narrowband flat fading channel model such that the $N \times 1$ channel vector for user $i$ can be written as $\mathbf{h}_{i}$, and the composite $N \times K$ channel matrix is denoted by $\mathbf{H}=\left[\mathbf{h}_{1} \mathbf{h}_{2} \ldots \mathbf{h}_{K}\right]$. We assume that the propagation channel is known to both the users and the cellular BS. This is an assumption in this study since we are concerned with analyzing the fundamental properties of a massive MIMO system using ray-based channels. In practice, reciprocity-based beamforming may be used where uplink channel estimates will be used for payload data transmission.

\section{A. Ray-based Channel Model}

In general, the propagation channel to user $i$ can be described as the superposition of many individual rays possibly arriving in clusters from a set of far-field scatterers. In simple terms, the channel is broken down into $P$ incident rays at the BS. ${ }^{2}$ Hence, for a ULA we have

$$
\mathbf{h}_{i}=\sum_{r=1}^{P} \gamma_{i r} \mathbf{a}\left(\phi_{i r}\right)
$$

where $\phi_{i r}$ is the azimuth angle of the $r^{\text {th }}$ ray, $\gamma_{i r}$ is a complex scaling factor for the magnitude and phase of the ray, and $\mathbf{a}\left(\phi_{i r}\right)$ is the $N \times 1$ array steering vector. In azimuth, the antenna array broadside is at $\phi_{i r}=0$, and end-fire is

\footnotetext{
${ }^{2}$ For ease of notation, we do not specifically itemize clusters, but the $P$ paths include any clustered rays. We note that since we are concerned with angular distributions of individual rays contributing to the channel impulse response, it is not necessary to categorize the channel model in terms of clusters.
} 
$\phi_{i r}= \pm \frac{\pi}{2}$. Common models for the scaling factor include random phase models [16], where $\gamma_{i r}=\sqrt{\beta_{i r}} \exp \left(j \Phi_{i r}\right), \beta_{i r}$ is the power of the $r^{\text {th }}$ ray and $\Phi_{i r}$ are i.i.d. $U[0,2 \pi]$ phase offsets. Hence, $\beta_{i}=\sum_{r=1}^{P} \beta_{i r}$ is the total link gain for user $i$. Also, complex Gaussian models have been proposed in [18], where $\gamma_{i r}=\sqrt{\beta_{i r}} u_{i r}$ and $u_{i r} \sim \mathcal{C N}(0,1)$. For both models, we note that $\mathbb{E}\left[\gamma_{i r}\right]=0, \mathbb{E}\left[\left|\gamma_{i r}\right|^{2}\right]=\beta_{i r}$ and $\mathbb{E}\left[\gamma_{i r}^{*} \gamma_{j s}\right]=0$ for all pairs $(i, r) \neq(j, s)$. For a ULA with normalized inter-element spacing $d$, measured in wavelengths, the steering vector is given by

$$
\mathbf{a}\left(\phi_{i r}\right)=\left[1, e^{2 \pi j d \sin \phi_{i r}}, e^{2 \pi j 2 d \sin \phi_{i r}}, \ldots, e^{2 \pi j(N-1) d \sin \phi_{i r}}\right]^{\mathrm{T}} .
$$

For a uniform planar array (UPA), the total number of antennas, $N$, is divided into $N_{x}$ and $N_{y}$ antennas in the $x$ and $y$ axes with inter-element spacings $d_{x}$ and $d_{y}$, respectively. The steering vectors can be represented by the Kronecker product of $\left(N_{x} \times 1\right) \mathbf{a}_{x}$ and $\left(N_{y} \times 1\right) \mathbf{a}_{y}$, which gives an $N_{x} N_{y} \times 1$ vector, $\mathbf{a}\left(\theta_{i r}, \phi_{i r}\right)=\mathbf{a}_{i r x} \otimes \mathbf{a}_{i r y}$. Note that

$$
\mathbf{a}_{i r x}=\left[1, e^{2 \pi j d_{x} \sin \theta_{i r} \cos \phi_{i r}}, \ldots, e^{2 \pi j\left(N_{x}-1\right) d_{x} \sin \theta_{i r} \cos \phi_{i r}}\right]^{\mathrm{T}},
$$

and

$$
\mathbf{a}_{i r y}=\left[1, e^{2 \pi j d_{y} \sin \theta_{i r} \sin \phi_{i r}}, \ldots, e^{2 \pi j\left(N_{y}-1\right) d_{y} \sin \theta_{i r} \sin \phi_{i r}}\right]^{\mathrm{T}} .
$$

Note that this definition of the steering vectors follows the notation in [20] where the array is defined in the $(x, y)$ plane. Hence, $\phi_{i r}$ is the angle of the $r^{\text {th }}$ ray for the $i^{\text {th }}$ user in the $(x, y)$-plane relative to the $x$-axis. The angle $\theta_{i r}$ is the angle of the $r^{\text {th }}$ ray for the $i^{\text {th }}$ user measured from the zenith direction ( $z$-axis). With this definition, if the UPA is horizontally oriented then $\phi_{i r}$ is an azimuth angle and $\theta_{i r}$ is an elevation angle. For vertically located arrays, the more general definition of the angles applies, where $\theta_{i r}$ and $\phi_{i r}$ are defined relative to the $\mathrm{z}$ and $\mathrm{x}$ axes, respectively. The $k^{\text {th }}$ elements of $\mathbf{a}_{i r x}$ and $\mathbf{a}_{i r y}$ are denoted by $\mathbf{a}_{i r x k}$ and $\mathbf{a}_{i r y k}$, respectively. In practice, each element has an active directional gain pattern, which attenuates the radiated power as a function of the steering direction. In order not to obfuscate the results and deviate focus from the asymptotic massive MIMO properties, we assume that each element has an equal gain in all directions (i.e., omni-directional), irrespective of the element location in the array.

\section{B. FP, Channel Hardening and Large System Potential}

Here, FP denotes asymptotic FP where $\mathbf{h}_{i}^{\mathrm{H}} \mathbf{h}_{j} / N \stackrel{\text { a.s. }}{\longrightarrow} 0$ as $N \rightarrow \infty$ [2]. Channel hardening refers to the property that $\mathbf{h}_{i}^{\mathrm{H}} \mathbf{h}_{i} / N \stackrel{\text { a.s. }}{\longrightarrow} \beta_{i}$ as $N \rightarrow \infty$, which is equivalent in our case to the definition in [4]. Now, FP and $\mathrm{CH}$ imply that the interference from one user to another vanishes relative to the signal power almost surely as $N \rightarrow \infty$. We extend this definition to the asymptotic regime where $N \rightarrow \infty, K \rightarrow \infty$ and $N / K \rightarrow \alpha$ (double-sided massive MIMO). Here, the equivalent question concerns whether the total interference power to user $i$ dominates the signal power. In order to investigate this behavior, we define

$$
\zeta_{\mathrm{LSP}}=\frac{\mathbb{E}\left[\left|\mathbf{h}_{i}^{\mathrm{H}} \mathbf{h}_{i}\right|^{2}\right]}{\sum_{j \neq i}^{K} \mathbb{E}\left[\left|\mathbf{h}_{i}^{\mathrm{H}} \mathbf{h}_{j}\right|^{2}\right]}=\frac{\mathbb{E}\left[\left|\mathbf{h}_{i}^{\mathrm{H}} \mathbf{h}_{i} / N\right|^{2}\right]}{\sum_{j \neq i}^{K} \mathbb{E}\left[\left|\mathbf{h}_{i}^{\mathrm{H}} \mathbf{h}_{j} / N\right|^{2}\right]} .
$$

Now, $\zeta_{\text {LSP }}$ is a fundamental performance metric, measuring the ratio of the desired mean channel power to the total mean interference power. We say that LSP holds if $\zeta_{\text {LSP }}$ converges to a positive constant as $N \rightarrow \infty$ and $N / K \rightarrow \alpha$. If $\mathrm{CH}$ holds, then $\mathbf{h}_{i}^{\mathrm{H}} \mathbf{h}_{i} / N$ converges to a positive constant and hence, the numerator of (2) also converges to a positive constant. Even when $\mathrm{CH}$ does not occur (see Sec. III-A), the expectation in the numerator of (2) will converge to a constant.

If $\mathrm{CH}$ holds then the numerator of (2) converges to a positive constant. Hence, LSP depends on the limiting behavior of the denominator of (2), namely $\mathbb{E}\left[\eta_{i}\right]$, the mean of the total normalized interference, where $\eta_{i}$ is defined as

$$
\eta_{i}=\sum_{j \neq i}^{K}\left|\mathbf{h}_{i}^{\mathrm{H}} \mathbf{h}_{j} / N\right|^{2} .
$$

Note that the limiting regime used for LSP, which supports double-sided massive MIMO, is far more challenging than traditional massive MIMO. In practice, the number of users will never grow without bound but the asymptotics are still useful in identifying the key properties of systems which are large in both $N$ and $K$.

\section{ULA: ChANNEL HARDENING, FP AND LSP}

$\mathrm{CH}, \mathrm{FP}$ and LSP are now considered for ray-based channels for a ULA, and let $\mathbf{a}_{i r}$ be the steering vector for user $i$, path $r, \mathbf{a}_{i r}=\mathbf{a}\left(\phi_{i r}\right)$ and the $k^{\text {th }}$ element of $\mathbf{a}_{i r}$ is denoted $\mathbf{a}_{i r k}$.

\section{A. Channel Hardening}

Consider the term, $\mathbf{h}_{i}^{\mathrm{H}} \mathbf{h}_{i} / N$, for a ULA. We have

$$
\begin{aligned}
\frac{\mathbf{h}_{i}^{\mathrm{H}} \mathbf{h}_{i}}{N} & =\frac{1}{N} \sum_{r=1}^{P} \gamma_{i r}^{*} \mathbf{a}_{i r}^{\mathrm{H}} \sum_{s=1}^{P} \gamma_{i s} \mathbf{a}_{i s} \\
& =\sum_{r=1}^{P}\left|\gamma_{i r}\right|^{2}+\frac{1}{N} \sum_{r=1, r \neq s}^{P} \sum_{s=1}^{P} \gamma_{i r}^{*} \gamma_{i s} \mathbf{a}_{i r}^{\mathrm{H}} \mathbf{a}_{i s}=X_{i}+E_{i},
\end{aligned}
$$

where $X_{i}=\sum_{r=1}^{P}\left|\gamma_{i r}\right|^{2}$ is independent of $N$. Thus the limiting value depends entirely on $\lim _{N \rightarrow \infty} E_{i}$, which in turn depends on $\lim _{N \rightarrow \infty} \mathbf{a}_{i r}^{\mathrm{H}} \mathbf{a}_{i s} / N$, where $r \neq s$. Now,

$$
\begin{aligned}
\left|\frac{\mathbf{a}_{i r}^{\mathrm{H}} \mathbf{a}_{i s}}{N}\right| & =\left|\frac{1}{N} \sum_{n=0}^{N-1} e^{-j 2 \pi d n \sin \phi_{i r}} e^{j 2 \pi d n \sin \phi_{i s}}\right|, \\
& =\frac{1}{N}\left|\frac{\sin (N \tau(i, i) / 2)}{\sin (\tau(i, i) / 2)}\right| \stackrel{\text { a.s. }}{\longrightarrow} 0,
\end{aligned}
$$

where $\tau(i, i)=2 \pi d\left[\sin \phi_{i s}-\sin \phi_{i r}\right]$, using simple results on geometric series. Almost sure convergence follows from the fact that convergence is guaranteed unless $\sin \phi_{i r}=\sin \phi_{i s}$, an event with probability zero for continuous angular variables. Thus, we have $\mathbf{h}_{i}^{\mathrm{H}} \mathbf{h}_{i} / N \stackrel{\text { a.s. }}{\longrightarrow} X_{i}$ as $N \rightarrow \infty$. Note that for random phase models, $X_{i}=\beta_{i}$ and traditional $\mathrm{CH}$ occurs where $\mathbf{h}_{i}^{\mathrm{H}} \mathbf{h}_{i} / N \stackrel{\text { a.s. }}{\longrightarrow} \beta_{i}$, a deterministic limit. In contrast, for complex Gaussian models, $\left|\gamma_{i r}\right|^{2}=\beta_{i r}\left|u_{i r}\right|^{2}$, which gives 
a random limit, as $X_{i}=\sum_{r=1}^{P}\left|\gamma_{i r}\right|^{2}$ is a weighted sum of exponential variables. Hence, we see that the existence of $\mathrm{CH}$ depends on the nature of the model for the ray coefficients. Note that the $\mathrm{CH}$ analysis in [22] was for arbitrary array topologies but relied on uniform angles. The ratio of sines in (5) appears frequently in the analysis and the more compact representation using the Dirichlet kernel is used in all subsequent derivations.

\section{B. FP (Single-Sided Massive MIMO)}

For FP, results are simple following the same methodology as for $\mathrm{CH}$. First, we write

$$
\frac{\mathbf{h}_{i}^{\mathrm{H}} \mathbf{h}_{j}}{N}=\frac{1}{N} \sum_{r=1}^{P} \sum_{s=1}^{P} \gamma_{i r}^{*} \gamma_{j s} \mathbf{a}_{i r}^{\mathrm{H}} \mathbf{a}_{j s},
$$

and then we use (5) to show that

$$
\begin{aligned}
\left|\frac{\mathbf{a}_{i r}^{\mathrm{H}} \mathbf{a}_{j s}}{N}\right| & =\frac{1}{N}\left|\frac{\sin (N \tau(i, j) / 2)}{\sin (\tau(i, j) / 2)}\right|, \\
& =\frac{2 \pi}{N}\left|D_{\frac{N-1}{2}}(\tau(i, j))\right| \stackrel{\text { a.s. }}{\longrightarrow} 0,
\end{aligned}
$$

as $N \rightarrow \infty$, where $\tau(i, j)=2 \pi d\left[\sin \phi_{j s}-\sin \phi_{i r}\right]$. Hence, FP is proven very simply for all ray-based models where $\sin \phi_{i r}=\sin \phi_{i s}$ has probability zero. A simple condition for this to hold is that the angles are continuous random variables, a property held by all proposed models. ${ }^{3}$ Therefore, FP, the key property enabling single-sided massive MIMO, holds for all ray distributions considered to date, such as uniform, wrapped Gaussian, and Laplacian.

\section{Large System Potential (Double-Sided Massive MIMO)}

We analyze LSP of ray-based channels in the limiting regime $K \rightarrow \infty, N \rightarrow \infty, N / K \rightarrow \alpha$.

1) Ray-based Models: The ratio $\zeta_{\text {LSP }}$ in (2) has a numerator satisfying $\mathbb{E}\left[\left|\mathbf{h}_{i}^{\mathrm{H}} \mathbf{h}_{i}\right|^{2} / N^{2}\right] \stackrel{\text { a.s. }}{\longrightarrow} \mathbb{E}\left[X_{i}^{2}\right]$ from Sec. III-A and $\mathbb{E}\left[X_{i}^{2}\right]$ is finite. Hence, LSP depends on the asymptotic properties of the denominator, $\mathbb{E}\left[\eta_{i}\right]$. Here, we write,

$$
\begin{aligned}
\mathbb{E}\left[\eta_{i}\right] & =\mathbb{E}\left[\frac{1}{N} \sum_{j=1, j \neq i}^{K} \frac{1}{N}\left|\sum_{r=1}^{P} \sum_{s=1}^{P} \gamma_{i r}^{*} \gamma_{j s} \mathbf{a}_{i r}^{\mathrm{H}} \mathbf{a}_{j s}\right|^{2}\right] \\
& =\frac{1}{N} \sum_{j=1, j \neq i}^{K} \sum_{r=1}^{P} \sum_{s=1}^{P} \mathbb{E}\left[\left|\gamma_{i r}\right|^{2}\right] \mathbb{E}\left[\left|\gamma_{j s}\right|^{2}\right] \frac{1}{N} \mathbb{E}\left[\left|\mathbf{a}_{i r}^{\mathrm{H}} \mathbf{a}_{j s}\right|^{2}\right], \\
& =\beta_{i}\left(\frac{\sum_{j=1, j \neq i}^{K} \beta_{j}}{N}\right) \mu_{\mathrm{ULA}},
\end{aligned}
$$

using the basic properties of the $\gamma_{i r}$ terms and the notation

$$
\begin{aligned}
\mu_{\mathrm{ULA}} & \triangleq \frac{1}{N} \mathbb{E}\left[\left|\mathbf{a}_{i r}^{\mathrm{H}} \mathbf{a}_{j s}\right|^{2}\right], \\
& =\frac{1}{N} \sum_{n=0}^{N-1} \sum_{m=0}^{N-1} \mathbb{E}\left[e^{j 2 \pi d(m-n) \sin \phi_{i r}}\right] \mathbb{E}\left[e^{j 2 \pi d(n-m) \sin \phi_{j s}}\right], \\
& =\frac{1}{N} \sum_{n=0}^{N-1} \sum_{m=0}^{N-1}\left|\mathbb{E}\left[e^{j 2 \pi d(m-n) \sin \phi_{i r}}\right]\right|^{2}
\end{aligned}
$$

\footnotetext{
${ }^{3}$ This was demonstrated in [20], but only for the case of uniform distribution. In contrast, the result in (7) is general.
}

This relies on the fact that the scaling factors are independent and azimuth angles are i.i.d random variables. Hence, the steering vectors in $\mu_{\mathrm{ULA}}$ are two generic but independent vectors and the subscripts $i r$ and $j s$ are not strictly necessary. Now, we set $\hat{\phi}_{i r}=2 \pi d \sin \phi_{i r}$, and rewrite (9) as

$$
\mu_{\mathrm{ULA}}=1+2 \sum_{q=1}^{N-1}\left(1-\frac{q}{N}\right)\left|\mathbb{E}\left[e^{-j q \hat{\phi}_{i r}}\right]\right|^{2} .
$$

In a typical drop of random user locations, the strong law of large numbers ensures that $\sum_{j=1}^{K} \beta_{j} / K$ converges to $\bar{\beta}$ as $K \rightarrow \infty$, where $\bar{\beta}$ is a finite mean power. Hence, we have $\lim _{N \rightarrow \infty} \mathbb{E}\left[\eta_{i}\right]=\left(\beta_{i} \bar{\beta} / \alpha\right) \lim _{N \rightarrow \infty}\left\{\mu_{\mathrm{ULA}}\right\}$. Hence, the asymptotic behavior of $\mathbb{E}\left[\eta_{i}\right]$ depends on $\mu_{\mathrm{ULA}}$ which in turn depends on how quickly $\left|\mathbb{E}\left[e^{-j q \hat{\phi}_{\text {ir }}}\right]\right|^{2}$ decays. In the following theorem we present a general answer to this question.

Theorem 1. The term $\mathbb{E}\left[e^{-j q \hat{\phi}_{i r}}\right]$ decays as $q^{-\frac{1}{2}}$ as $q \rightarrow \infty$ with the asymptotic representation:

$$
\begin{aligned}
& \mathbb{E}\left[e^{-j q \hat{\phi}_{i r}}\right] \sim \\
& \frac{1}{\sqrt{d q}}\left(f_{\phi}\left(-\frac{\pi}{2}\right) e^{j\left(2 \pi d q-\frac{\pi}{4}\right)}+f_{\phi}\left(\frac{\pi}{2}\right) e^{-j\left(2 \pi d q-\frac{\pi}{4}\right)}\right),
\end{aligned}
$$

where $f_{\phi}(\cdot)$ is the probability density function (PDF) of $\phi_{i r}$.

Proof. The proof is given in Appendix A.

2) Implications of Theorem 1: Equation (11) in Theorem 1 is a remarkable result with a simple and intuitive interpretation, wide generality and important implications for massive MIMO:

- In terms of generality, (11) only requires the angular PDF, $f_{\phi}(\cdot)$, not to have singularities which are worse than $O\left(x^{-1 / 2}\right)$ at $x=0$. This covers all proposed models. Certainly, all proposed models thus far are continuous, so this condition is easily satisfied.

- Interpreting (11) we see that if the end-fire direction has no energy, $f_{\phi}( \pm \pi / 2)=0$, then $\mathbb{E}\left[e^{-j q \hat{\phi}_{i r}}\right]=$ 0 . Alternatively, if some end-fire radiation occurs then $f_{\phi}( \pm \pi / 2)>0$ and $\mathbb{E}\left[e^{-j q \hat{\phi}_{i r}}\right]=O\left(q^{-1 / 2}\right)$. From the above, it follows that if there is no end-fire radiation, $\mu_{\mathrm{ULA}}$ is finite and the mean interference cannot dominate the mean power of the desired channel.

- Further, if there is end-fire radiation, then LSP does not hold as $\mu_{\mathrm{ULA}} \rightarrow \infty$. This conclusion holds by inspection of (10). When $\mathbb{E}\left[e^{-j q \hat{\phi}_{i r}}\right]$ is $O\left(q^{-1 / 2}\right)$, then $\sum_{q=1}^{N}\left|\mathbb{E}\left[e^{-j q \hat{\phi}_{i r}}\right]\right|^{2}$ is $O(\log N)$ using well known properties of the series $\sum_{q=1}^{N} \frac{1}{q}$. Also, $\sum_{q=1}^{N} \frac{q}{N}\left|\mathbb{E}\left[e^{-j q \hat{\phi}_{i r}}\right]\right|^{2}$ is finite, so that $\mu_{\mathrm{ULA}}$ grows to infinity, but at a very slow logarithmic rate. ${ }^{4}$ Note that this interference growth can be described as critical as $\mathbb{E}\left[e^{-j q \hat{\phi}_{i r}}\right]$ decays at exactly the critical rate $\left(q^{-1 / 2}\right)$ required for logarithmic growth. Any reduction at all in the decay rate would deliver finite interference and therefore would enable LSP to hold.

${ }^{4}$ Note that logarithmic growth always refers to growth that is logarithmic in $N$, the number of BS antennas. 
- The importance of the end-fire direction can be understood as follows. For a ULA, it is not the proximity of two ray angles that drives the interference, but the difference in the sines of the angles (see $\tau(i, j)$ in (7)). For angles close to broadside the difference in sines is largest, while near end-fire the difference is smallest, resulting in greater interference.

- We note that LSP does hold for i.i.d. Rayleigh fading channels. To see this we use the results in [6] to give $\zeta_{\text {LSP }}=\beta_{i} \bar{\beta} / \alpha$. Hence, ray-based models differ from classical statistical channel models in this regard. Note that the result in [6] is not given explicitly but can be obtained from Table I in [6].

- Overall, the result in (11) is extremely positive for double-sided massive MIMO. We have shown that in the challenging scenario where both $K$ and $N$ grow large, the interference, relative to the power of the desired channel, grows very slowly (logarithmically). Also, the scaling of this growth factor is very small, since a large amount of end-fire radiation is unlikely. Practical deployments typically employ patch elements with average look angles on the order of $+/-45^{\circ}$ (designed according to the downtilt angle of the array as well as the environment) [27]. This greatly attenuates the end-fire (or near by) radiation since the patterns create explicit nulls to reject the incoming wavefronts in those directions.

Given the power of these results, it is useful to validate the conclusions with some closed-form special cases. Note that although uniform and von-Mises (VM) angular distributions are not derived from measurements in real environments they are useful, both for validating Theorem 1 and providing exact asymptotics which are not perturbed by simulation error.

3) Special Cases: Uniform Distribution: When $\phi_{i r} \sim$ $U[0,2 \pi], f_{\phi}(x)=\frac{1}{2 \pi}$ for $-\pi \leq x \leq \pi$ and (11) becomes

$$
\mathbb{E}\left[e^{-j q \hat{\phi}_{i r}}\right] \sim \frac{1}{\pi \sqrt{d q}} \cos \left(2 \pi d q-\frac{\pi}{4}\right) .
$$

This limiting value is verified in the uniform case where the exact solution is known as $\mathbb{E}\left[e^{-j q \hat{\phi}_{i r}}\right]=\mathbb{E}\left[e^{-j q 2 \pi d \sin \phi_{i r}}\right]=$ $J_{0}(2 \pi d q)$ [26, p. 375]. For large values of $q, J_{0}(2 \pi d q) \sim$ $\cos \left(2 \pi d q-\frac{\pi}{4}\right) / \pi \sqrt{d q}$ [26, p. 364], which agrees with (11). Hence, the general asymptotic analysis in (11) is supported and the exact value of $\mathbb{E}\left[e^{-j q \hat{\phi}_{i r}}\right]$ can be used in (10) to give the exact value of $\mu_{\mathrm{ULA}}$.

4) Special Cases: Von-Mises Distribution: The VM distribution has also been used in angular modeling [28] and has the PDF given by

$$
f_{\phi}(x)=\frac{e^{\kappa \cos (x-\mu)}}{2 \pi I_{0}(\kappa)},-\pi \leq x \leq \pi,
$$

where $\mu$ is a measure of location and $\kappa$ is a measure of concentration. Substituting into (11),

$\mathbb{E}\left[e^{-j q \hat{\phi}_{i r}}\right] \sim \frac{e^{j 2 \pi d q}}{\sqrt{d q}}\left(\frac{e^{\kappa \cos \left(-\frac{\pi}{2}-\mu\right)}}{2 \pi I_{0}(\kappa)} e^{-j \frac{\pi}{4}}+\frac{e^{\kappa \cos \left(\frac{\pi}{2}-\mu\right)}}{2 \pi I_{0}(\kappa)} e^{j \frac{\pi}{4}}\right)$.
The exact solution can be found by integration, giving

$$
\mathbb{E}\left[e^{-j q \hat{\phi}_{i r}}\right]=\frac{I_{0}\left(\sqrt{\kappa^{2} \cos ^{2}(\mu)+(\kappa \sin (\mu)-j 2 \pi d q)^{2}}\right)}{I_{0}(\kappa)} .
$$

Further analysis shows that (15) is asymptotically equal to (14). This is briefly explained as follows. For large $q$, the argument of $I_{0}(\cdot)$ in the numerator of (15) is approximately $\kappa \sin (\mu)-j 2 \pi d q$. Then we use the large argument approximation of $I_{0}(\kappa \sin (\mu)-j 2 \pi d q)$ [26, p. 364] and simplify to give (13). Hence, for the VM case also, we have verified (11) and given an exact solution for $\mathbb{E}\left[e^{-j q \hat{\phi}_{i r}}\right]$.

\section{Avoiding Interference Growth}

Since the logarithmic interference growth predicted by Theorem 1 is critical, it can be removed by simple methods. For example, for any finite $K$, LSP exists and for the asymptotic case, where $K$ is increasing, LSP can be assured by trivial scheduling methods based on user separation. This is shown in the following.

Expanding $\eta_{i}=\sum_{j \neq i}^{K}\left|\mathbf{h}_{i}^{\mathrm{H}} \mathbf{h}_{j} / N\right|^{2}$ in terms of the steering vectors gives

$$
\eta_{i}=\sum_{j \neq i} \frac{1}{N^{2}} \sum_{r, s} \sum_{r^{\prime}, s^{\prime}} \gamma_{i r}^{*} \gamma_{j s} \gamma_{i r^{\prime}} \gamma_{j s^{\prime}}^{*} \mathbf{a}_{i r}^{\mathrm{H}} \mathbf{a}_{i s} \mathbf{a}_{j s^{\prime}}^{\mathrm{H}} \mathbf{a}_{i r^{\prime}} .
$$

From (5), $\eta_{i}$ can be rewritten as

$$
\begin{aligned}
\eta_{i}= & \sum_{j \neq i} \sum_{r, s, r^{\prime}, s^{\prime}} \Gamma_{i j r s r^{\prime} s^{\prime}}\left(\frac{2 \pi}{N}\right)^{2} \\
& \times\left|D_{\frac{N-1}{2}}\left(\tau_{1}(i, j)\right)\right|\left|D_{\frac{N-1}{2}}\left(\tau_{2}(i, j)\right)\right|,
\end{aligned}
$$

where $\sum_{r, s, r^{\prime}, s^{\prime}}=\sum_{r, s} \sum_{r^{\prime}, s^{\prime}}$ and $\Gamma_{i j r s r^{\prime} s^{\prime}}=$ $\gamma_{i r}^{*} \gamma_{j s} \gamma_{i r^{\prime}} \gamma_{j s^{\prime}}^{*}, \tau_{1}(i, j)=2 \pi d\left[\sin \phi_{j s}-\sin \phi_{i r}\right]$ and $\tau_{2}(i, j)=$ $2 \pi d\left[\sin \phi_{j s^{\prime}}-\sin \phi_{i r^{\prime}}\right]$. Note that $\sum_{r, s, r^{\prime}, s^{\prime}} \Gamma_{i j r s r^{\prime} s^{\prime}}$ is finite and for $\tau_{u}(i, j) \neq 0,\left|\frac{\sin \left(N \tau_{u}(i, j) / 2\right)}{\sin \left(\tau_{u}(i, j) / 2\right)}\right|$ is bounded for $u=1,2$. Hence, for finite $K, \eta_{i} \rightarrow 0$ for $\tau_{1}(i, j) \neq 0$, $\tau_{2}(i, j) \neq 0$ as the number of terms in the sum is equal to $K-1$ but the summand is $O\left(N^{-2}\right)$. Since $P\left(\tau_{1}(i, j)=\right.$ $0)=P\left(\tau_{2}(i, j)=0\right)=0$ for continuous angular variables, it follows that $\eta_{i} \stackrel{\text { a.s. }}{\longrightarrow} 0$ and LSP holds.

Next, consider the asymptotic case where the number of users $(N / K=\alpha)$ is growing but users are only scheduled together if the sines of their ray angles are separated by more than a given protection level, $\epsilon>0$. Hence, $\left|\tau_{u}(i, j)\right|>2 \pi d \epsilon$. Using this inequality in (17) gives

$$
\eta_{i} \leq \frac{1}{N^{2} \sin ^{2}(\pi d \epsilon)} \sum_{j \neq i} \sum_{r, s, r^{\prime}, s^{\prime}} \Gamma_{i j r s r^{\prime} s^{\prime}} .
$$

Since the right side of (18) converges to zero as $N \rightarrow \infty$ it follows that $\eta_{i} \stackrel{\text { a.s. }}{\longrightarrow} 0$ and LSP holds.

\section{UPA: CHANNEL HARDENING, FP AND LSP}

In this section, we extend the ULA results on $\mathrm{CH}, \mathrm{FP}$ and LSP to a UPA. Throughout the work on LSP for a UPA we assume that $N_{x} \rightarrow \infty$ and $N_{y} \rightarrow \infty$ as $N \rightarrow \infty$. 


\section{A. Channel Hardening}

The $\mathrm{CH}$ results presented in (4) for a ULA remain valid for any array structure. Hence, $\mathbf{h}_{i}^{\mathrm{H}} \mathbf{h}_{i} / N \stackrel{\text { a.s. }}{\longrightarrow} X_{i}$ if $\mathbf{a}_{i r}^{\mathrm{H}} \mathbf{a}_{i s} \stackrel{\text { a.s. }}{\longrightarrow} 0$. For the UPA we have

$$
\begin{aligned}
\frac{\mathbf{a}_{i r}^{\mathrm{H}} \mathbf{a}_{i s}}{N} & =\frac{1}{N}\left(\mathbf{a}_{i r x} \otimes \mathbf{a}_{i r y}\right)^{\mathrm{H}}\left(\mathbf{a}_{i s x} \otimes \mathbf{a}_{i s y}\right), \\
& =\frac{1}{N}\left(\mathbf{a}_{i r x}^{\mathrm{H}} \mathbf{a}_{i s x}\right)\left(\mathbf{a}_{i r y}^{\mathrm{H}} \mathbf{a}_{i s y}\right),
\end{aligned}
$$

from basic properties of Kronecker products. Now, the cross products $\mathbf{a}_{i r x}^{\mathrm{H}} \mathbf{a}_{i s x}$ and $\mathbf{a}_{i r y}^{\mathrm{H}} \mathbf{a}_{i s y}$ have a similar form to the ULA. Hence, from (4) we can deduce that

$$
\left|\frac{\mathbf{a}_{i r}^{\mathrm{H}} \mathbf{a}_{i s}}{N}\right|=\frac{2 \pi}{N_{x}}\left|D_{\frac{N_{x}-1}{2}}\left(\tau_{x}(i, i)\right)\right| \frac{2 \pi}{N_{y}}\left|D_{\frac{N_{y}-1}{2}}\left(\tau_{y}(i, i)\right)\right|,
$$

where $\tau_{x}(i, i)=2 \pi d_{x}\left(\sin \theta_{i s} \cos \phi_{i s}-\sin \theta_{i r} \cos \phi_{i r}\right)$ and $\tau_{y}(i, i)=2 \pi d_{y}\left(\sin \theta_{i s} \sin \phi_{i s}-\sin \theta_{i r} \sin \phi_{i r}\right)$. As in Sec. III-A, $\mathbf{a}_{i r}^{\mathrm{H}} \mathbf{a}_{i s} / N$ converges to zero unless $\tau_{x}(i, i)=0$ or $\tau_{y}(i, i)=0$, an event with probability zero. Hence $\mathbf{a}_{i r}^{\mathrm{H}} \mathbf{a}_{i s} / N \stackrel{\text { a.s. }}{\longrightarrow} 0$ and $\mathbf{h}_{i}^{\mathrm{H}} \mathbf{h}_{i} / N \stackrel{\text { a.s. }}{\longrightarrow} X_{i}$.

\section{B. FP (Single-Sided Massive MIMO)}

The ULA results in (6) and (7) show that for any array, FP occurs if $\frac{\mathbf{a}_{i r}^{\mathrm{H}} \mathbf{a}_{j s}}{N} \stackrel{\text { a.s. }}{\longrightarrow} 0$ as $N \rightarrow \infty$. Following the same calculation as in (20) gives

$$
\left|\frac{\mathbf{a}_{i r}^{\mathrm{H}} \mathbf{a}_{j s}}{N}\right|=\frac{2 \pi}{N_{x}}\left|D_{\frac{N_{x}-1}{2}}\left(\tau_{x}(i, j)\right)\right| \frac{2 \pi}{N_{y}}\left|D_{\frac{N_{y}-1}{2}}\left(\tau_{y}(i, j)\right)\right|,
$$

where $\tau_{x}(i, j)=2 \pi d_{x}\left(\sin \theta_{j s} \cos \phi_{j s}-\sin \theta_{i r} \cos \phi_{i r}\right)$ and $\tau_{y}(i, j)=2 \pi d_{y}\left(\sin \theta_{j s} \sin \phi_{j s}-\sin \theta_{i r} \sin \phi_{i r}\right)$. Since $P\left(\tau_{x}(i, j)=0\right)=P\left(\tau_{y}(i, j)=0\right)=0$ for continuous angular variables, it follows that $\eta_{i} \stackrel{\text { a.s. }}{\longrightarrow} 0$ and FP holds.

\section{Large System Potential (Double-Sided Massive MIMO)}

The existence of LSP for a UPA depends on whether the expression $\mu_{\mathrm{UPA}}=\frac{1}{N} \mathbb{E}\left[\left|\mathbf{a}_{i r}^{\mathrm{H}} \mathbf{a}_{j s}\right|^{2}\right]$ converges or not. This is analyzed in Theorem 2 .

Theorem 2. The term $\mu_{U P A}$ for a UPA antenna structure grows logarithmically with the following representation:

$$
\mu_{U P A}=\sum_{v=1-N_{x}}^{N_{x}-1} \sum_{w=1-N_{y}}^{N_{y}-1}\left(1-\frac{|v|}{N_{x}}\right)\left(1-\frac{|w|}{N_{y}}\right)\left|M_{v, w}\right|^{2},
$$

where $M_{v, w}=\mathbb{E}\left[e^{-j 2 \pi \nu_{v, w} \sin (\theta) \cos \left(\phi-\Delta_{v, w}\right)}\right], \quad \nu_{v, w}=$ $\sqrt{v^{2} d_{x}^{2}+w^{2} d_{y}^{2}}$, and $\Delta_{v, w}=\tan ^{-1}\left(\frac{w d_{y}}{v d_{x}}\right)$.

Proof. The proof is given in Appendix B.

1) Implications of Theorem 2: As in the ULA case, App. B shows that the interference growth is critical and any reduction in the rate of interference accumulation will lead to LSP holding. The result holds for any continuous angular distributions so is extremely general. Note that a ULA would normally aim to null the end-fire direction (see (11)) and perfect nulling would avoid the interference growth. In contrast, a similar argument using (46) shows that $f_{\theta}(x)$ must equal zero for $x$ in $\{-\pi / 2,0, \pi / 2\}$ in order to avoid interference growth. For a vertical UPA, to null the broadside direction is clearly unsuitable as it requires nulling the dominant azimuth plane $(\theta=0)$. In general for all types of UPA, a more symmetric structure means that there are no sets of special angles which avoid interference growth and for which the radiation is unwanted. Since the proof is complex, it is instructive to look at the uniform case where a closed-form result for $\mu_{\mathrm{UPA}}$ can be derived and shown to grow logarithmically as in Theorem 2. This is shown in the following.

2) Special Case: Uniform Distribution: We derive a closedform equation for $\mu_{\mathrm{UPA}}$ in the simplest uniform case, where the azimuth angle is $\phi_{i r} \sim U[0,2 \pi]$ and the elevation angle is $\theta_{i r} \sim U\left[-\frac{\pi}{2}, \frac{\pi}{2}\right]$. In this scenario, the following result applies.

Result. The value of $\mu_{U P A}$ for angles uniform in azimuth and in elevation is given by

$$
\begin{aligned}
\mu_{U P A} & =\frac{1}{N} \sum_{r_{x}=1}^{N_{x}} \sum_{r_{y}=1}^{N_{y}} \sum_{s_{x}=1}^{N_{x}} \sum_{s_{y}=1}^{N_{y}} \\
& \times J_{0}^{4}\left(\pi \sqrt{d_{y}^{2}\left(r_{y}-s_{y}\right)^{2}+d_{x}^{2}\left(r_{x}-s_{x}\right)^{2}}\right) .
\end{aligned}
$$

where the proof is given in Appendix C. In Appendix D, this result is used to demonstrate the logarithmic growth of $\mu_{\mathrm{UPA}}$.

3) Avoiding Interference Growth: As for the ULA, interference growth can be avoided by using finite $K$ or simple scheduling. This is shown in the following. For a UPA, substituting the associated steering vectors into (16) gives

$$
\begin{aligned}
\eta_{i} & =\sum_{j \neq i} \frac{1}{N^{2}} \sum_{r, s, r^{\prime}, s^{\prime}} \Gamma_{i j r s r^{\prime} s^{\prime}} \mathbf{a}_{i r x}^{\mathrm{H}} \mathbf{a}_{i s x} \mathbf{a}_{i r y}^{\mathrm{H}} \mathbf{a}_{i s y} \mathbf{a}_{j s^{\prime} x}^{\mathrm{H}} \mathbf{a}_{i r^{\prime} x} \\
& \times \mathbf{a}_{j s^{\prime} y}^{\mathrm{H}} \mathbf{a}_{i r^{\prime} y} .
\end{aligned}
$$

As in (20), all of the four cross products of steering vectors in $x$ and $y$ domains have representations as ratios of sine functions. Hence,

$$
\begin{aligned}
\eta_{i} & =\sum_{j \neq i} \sum_{r, s, r^{\prime}, s^{\prime}} \frac{16 \pi^{4}}{N^{2}} \Gamma_{i j r s r^{\prime} s^{\prime}}\left|D_{\frac{N_{x}-1}{2}}\left(\tau_{x}(i, j)\right)\right| \\
& \times\left|D_{\frac{N_{y}-1}{2}}\left(\tau_{y}(i, j)\right)\right|\left|D_{\frac{N_{x}-1}{2}}\left(\tau_{x}^{\prime}(i, i)\right)\right| \\
& \times\left|D_{\frac{N_{y}-1}{2}}\left(\tau_{y}^{\prime}(j, j)\right)\right|,
\end{aligned}
$$

where $\tau_{x}(i, j)$ and $\tau_{y}(i, j)$ are defined in Sec. IV-B, $\tau_{x}^{\prime}(i, i)=2 \pi d_{x}\left(\sin \theta_{i s^{\prime}} \cos \phi_{i s^{\prime}}-\sin \theta_{i r^{\prime}} \cos \phi_{i r^{\prime}}\right)$ and $\tau_{y}^{\prime}(j, j)=$ $2 \pi d_{y}\left(\sin \theta_{j s^{\prime}} \sin \phi_{j s^{\prime}}-\sin \theta_{j r^{\prime}} \sin \phi_{j r^{\prime}}\right)$. As before, for finite $K$ and $N$ growing, $\eta_{i} \rightarrow 0$ unless at least one of $\tau_{x}(i, j), \tau_{y}(i, j)$, $\tau_{x}^{\prime}(i, i), \tau_{y}^{\prime}(j, j)$ is zero, an event with probability 0 . Hence, $\eta_{i} \stackrel{\text { a.s. }}{\longrightarrow} 0$ as $N \rightarrow \infty$ so that LSP holds. In the asymptotic case where $K \rightarrow \infty$, considering scheduling using a similar protection threshold as used in Sec. III-D where users are only selected if $\min \left(\tau_{x}(i, j), \tau_{y}(i, j), \tau_{x}^{\prime}(i, i), \tau_{y}^{\prime}(j, j)\right)>2 \pi d \epsilon$. 
With this approach, we see that

$$
\eta_{i}<\sum_{j \neq i}^{K} \frac{1}{N^{2} \sin ^{4}(\pi d \epsilon)} \sum_{r, s, r^{\prime}, s^{\prime}} \Gamma_{i j r s r^{\prime} s^{\prime}} .
$$

Since the right side of (26) converges to zero as $N \rightarrow \infty$ $(N / K=\alpha), \eta_{i} \stackrel{\text { a.s. }}{\longrightarrow} 0$ and LSP holds.

\section{NuMERicAl RESUlts}

In Fig. 1 we demonstrate the $\mathrm{CH}$ and FP results for the ULA discussed in Sec. III for $K=2$ and an increasing number of antennas. We adopt the non-line-of-sight (NLOS) 3GPP angular and cluster parameters in [16]. The number of clusters is $C=20$, and the number of subpaths per cluster is $L=20$. Referring to the channel model in (1), $P=C L$. Each subpath angle of arrival (AoA) is modeled by a central cluster angle with a Gaussian distribution (zero mean and a standard deviation of $76.5^{\circ}$ ) plus a subray offset angle which is Laplacian with a standard deviation of $15^{\circ}$. We assume $\beta_{1}=\beta_{2}=1$ and subrays with equal powers. ${ }^{5}$ From the upper plot of Fig. 1, we see that the normalized power in the desired channel, $S=\left|\mathbf{h}_{i}^{\mathrm{H}} \mathbf{h}_{i}\right| / N \approx 1$ for large numbers of antennas. Similarly, the lower plot shows the mean of interference term, $I=\left|\mathbf{h}_{i}^{\mathrm{H}} \mathbf{h}_{j}\right| / N$ decreasing to zero as $N \rightarrow \infty$. Note that Fig. 1 plots simulations of $\mathbb{E}[I]$ for the ray-based model and analytical values of $\mathbb{E}[I]$ for i.i.d. Rayleigh fading (where each element of $\mathbf{H}$ is an independent $\mathcal{C N}(0,1)$ random variable) so that the variations do not obscure the trend. As expected, the convergence to FP is slower for the ray-based model but the initial rate of convergence is similar for both channels. Hence, both $\mathrm{CH}$ and FP are shown to occur for a typical parameter set as predicted by the analysis. Fig. 1 shows $\mathrm{CH}$ and FP occurring for a clustered channel model with wrapped Gaussian central cluster angles and Laplacian offsets. This numerical example is useful as it verifies the analysis for a commonly used raybased model structure. The analysis goes much further and proves the existence/non-existence of $\mathrm{CH}$ and the existence of FP for all ray-based of the form in (1) for a comprehensive range of ray distributions. These observations are in line with the $\mathrm{CH}$ measurements reported in [29].

Fig. 2 shows the power of the desired channel will either converge to a constant or a random variable, verifying the analysis in Sec. III-A. We assume the same model as in Fig. 1 but with two possibilities for the ray coefficients, $\gamma_{i r}$. The Akdeniz model [18] uses a complex Gaussian variable for $\gamma_{i r}$, while the 3GPP model [16] uses a random phase. As shown in Fig. 2, as the number of antennas grows, the cumulative distribution function (CDF) of the normalized desired channel power, $S=\mathbf{h}_{i}^{\mathrm{H}} \mathbf{h}_{i} / N$, with the Akdeniz model remains almost the same, indicating convergence to a random variable. In contrast, with the random phase model of 3GPP the CDF converges to a step function indicating that $S$ converges to a constant. Hence, as shown in Sec. III-A, CH can occur for ray-based channels, depending on the ray coefficient models.

\footnotetext{
${ }^{5}$ Equal ray powers are adopted for simplicity in Fig. 1 and Fig. 2 for initial verification of the $\mathrm{FP}$ and $\mathrm{CH}$ results, $\beta_{i r}=1 / C L$, and phases are uniformly distributed, $\phi_{i r} \sim U[0,2 \pi]$.
}
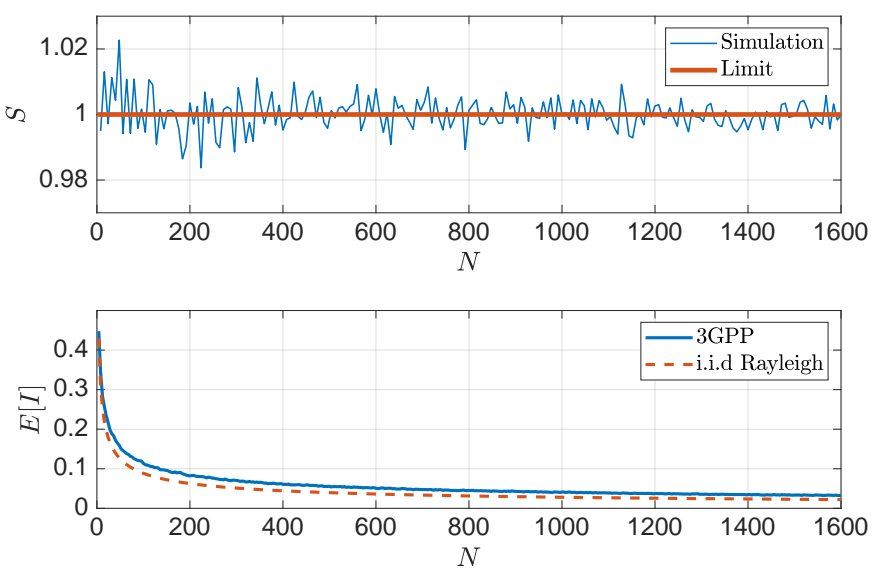

Fig. 1. Channel hardening and FP (3GPP angular parameters).

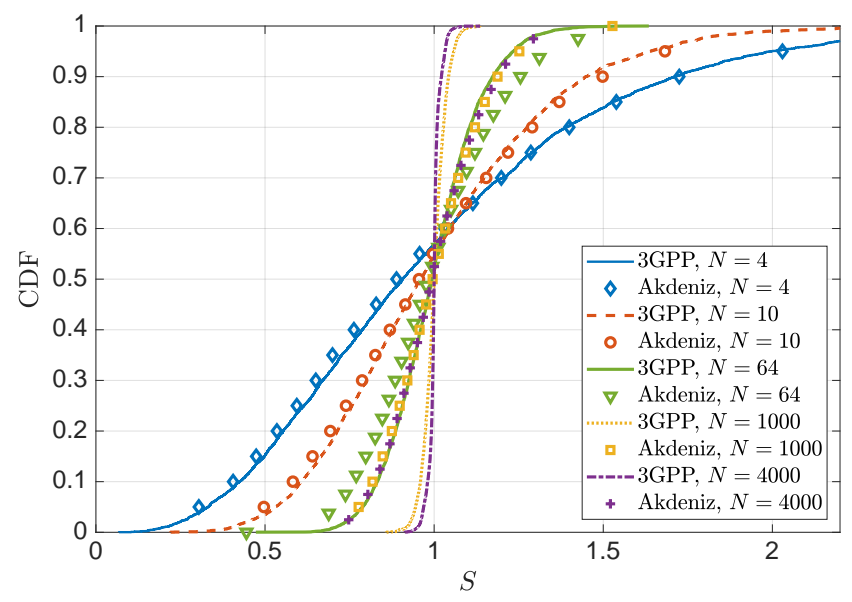

Fig. 2. Channel hardening for two types of channel models.

In Fig. 3, we show both the simulated and analytical results for $\mu_{\mathrm{ULA}}$ with uniform and VM distributions using the results in Secs. III-C3 and III-C4. We also show simulated values of $\mu_{\mathrm{ULA}}$ by adopting the angular parameters of the $3 \mathrm{GPP}$ model in [16] as in Fig. 1. The number of antennas and users are growing at the same ratio $N / K=\alpha=2$, while $\phi_{i r} \sim U[0,2 \pi]$ for the uniform model and $\kappa=4.23$ (for $30^{\circ}$ angle spread) and $\mu=0$ for VM. From Fig. 3 we see that the analysis agrees well with simulation for both uniform and VM models. We also note that the growth rates of $\mu_{\mathrm{ULA}}$ are different for all three models, due to the differences in the AoA distributions. Next, we give more details of the growth rate with regard to angular distributions.

In Fig. 4, we demonstrate the logarithmic growth rate of $\mu_{\mathrm{ULA}}$ against the number of antennas, $N(\alpha=2)$, for VM and uniform models with parameters as shown in the legend. The analytical results in Secs. III-C3 and III-C4 were used in generating $\mu_{\mathrm{ULA}}$ for the uniform and $\mathrm{VM}$ distributions, respectively. Although the analysis in Theorem 1 predicted logarithmic growth for $\mu_{\mathrm{ULA}}$, this is hard to verify from Fig. 3 . Hence, we substitute (11) into (10) and identify the dominant component of $\mu_{\mathrm{ULA}}$ giving $\mu_{\mathrm{ULA}} \sim m_{\text {slope }} \log (N)+C_{0}$, where 


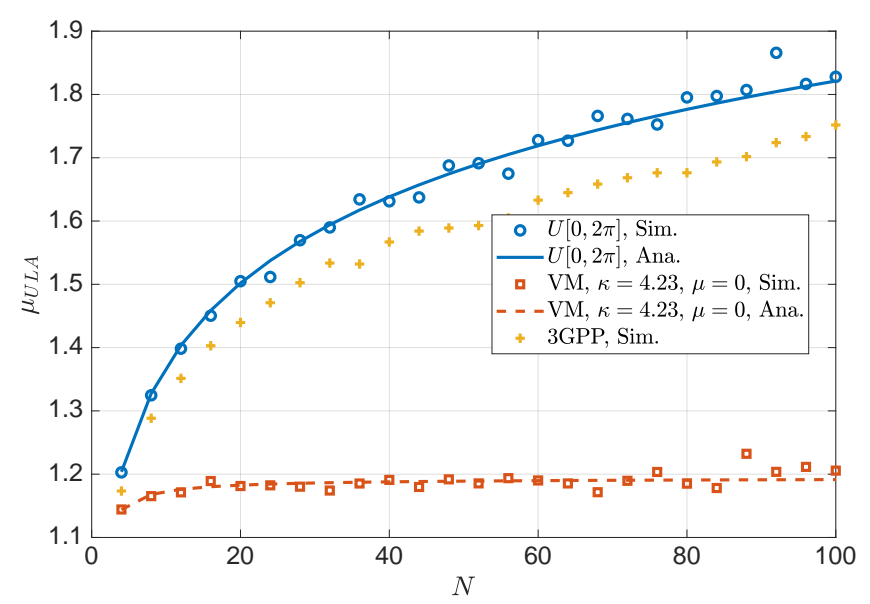

Fig. 3. $\mu_{\text {ULA }}$ vs $N$ for three different angular distributions.

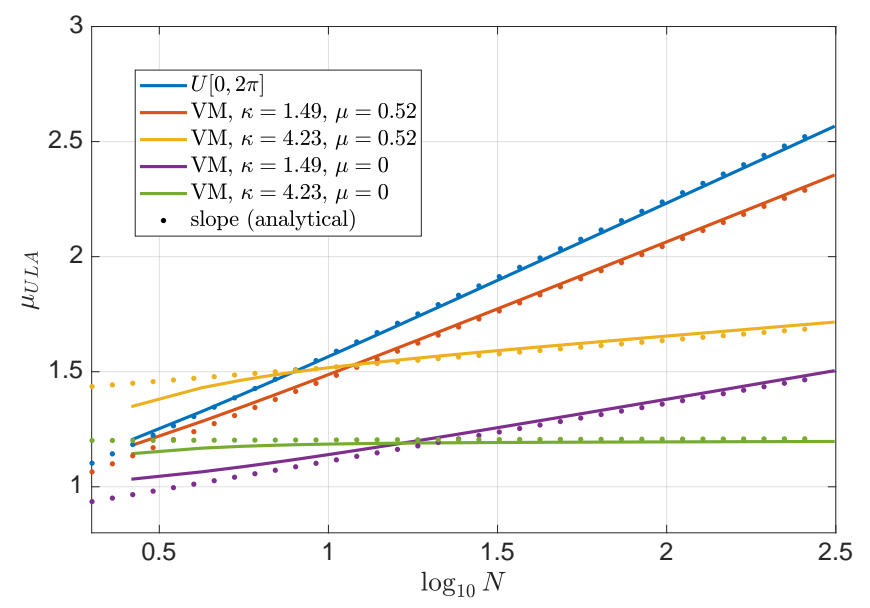

Fig. 4. Logarithmic growth of $\mu_{\mathrm{ULA}}$.

$C_{0}$ is a constant and

$$
m_{\text {slope }}=\frac{2\left(f_{\phi}^{2}\left(\frac{\pi}{2}\right)+f_{\phi}^{2}\left(-\frac{\pi}{2}\right)\right)}{d} .
$$

Hence, $m_{\text {slope }}$ determines how quickly $\mu_{\mathrm{ULA}}$ will grow. The uniform distribution has the highest interference growth rate, which is $m_{\text {slope }}^{\text {uniform }}=\left(\pi^{2} d\right)^{-1}$. For the VM model, the slope depends on $\kappa$ and $\mu$. In Fig. 4 we observe that $\mu_{\mathrm{ULA}}$ is clearly logarithmic in $N$, as predicted, and that the slope is correctly identified by (11), as shown by the dotted lines which have slope $m_{\text {slope }}$.

As well as verifying the logarithmic growth, Fig. 4 demonstrates some interesting angular properties. For both $\kappa=4.23$ (angle spread $=30^{\circ}$ ) and $\kappa=1.49$ (angle spread $=60^{\circ}$ ), $\mu_{\mathrm{ULA}}$ decreases as $\mu$ is reduced from $\mu=0.52\left(30^{\circ}\right)$ to $\mu=0$. This is because shifting the mean towards broadside reduces the interference inflation that occurs near end-fire. Secondly, for both $\mu=0$ and $\mu=0.52$ there is a crossover as $N$ increases. For small $N$, increased angular spread is beneficial as it spreads the rays and reduces the chance of high interference caused by rays in close proximity. However, for high $N$ the higher angular spread puts more probability near end-fire and this begins to dominate and causes higher interference.

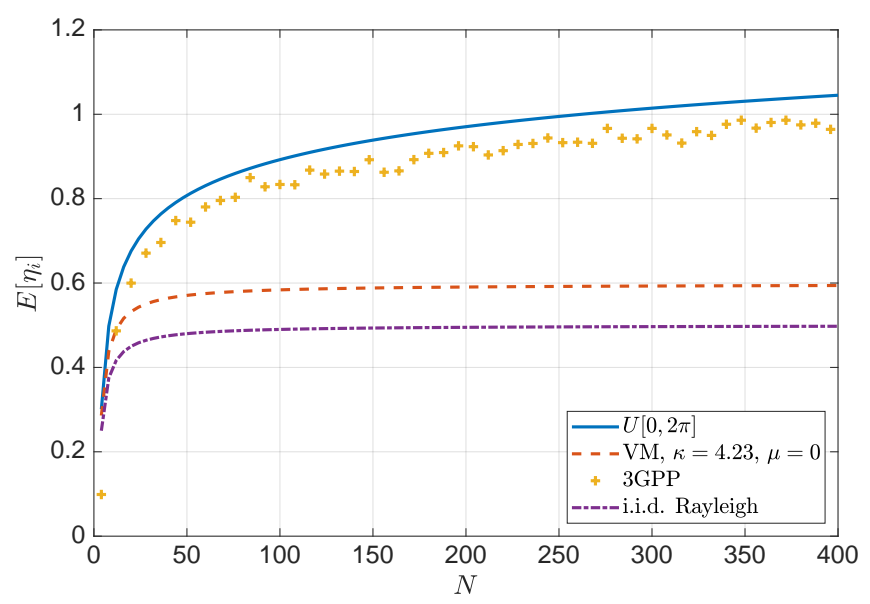

Fig. 5. $\mathbb{E}\left[\eta_{i}\right]$ vs $N$ for three angular distributions for ULA and Rayleigh fading.

In Fig. 5, we confirm via simulation for the 3GPP parameters and via analysis for the uniform and VM models that the mean global interference term, $\mathbb{E}\left[\eta_{i}\right]$, grows logarithmically as predicted by the analysis in Sec. III-C. For the uniform case, $\phi_{i r} \sim U[0,2 \pi]$, for $\mathrm{VM}, \kappa=4.23, \mu=0$, and for $3 \mathrm{GPP}$ we use the parameters considered in Fig. 1. For the uniform and VM models all user link gains and ray powers are equal, $\beta_{i r}=(C L)^{-1}$. For the 3GPP parameters, we also consider unequal ray powers and unequal user link gains. To avoid the substantial extra variation caused by shadowing models with large arrays we employ a simple deterministic model for these powers. The link gains decay exponentially from user 1 to user $K$ such that $\beta_{K}=\frac{1}{10} \beta_{1}$ and the cluster powers behave similarly. The desired user is then randomly allocated one of the $K$ distinct link gains $(\alpha=2)$. The levels are then adjusted to give the same total power as in the uniform and VM models and subrays in a particular cluster all have the same power as assumed in [30]. Fig. 5 shows the same logarithmic growth as Fig. 3, confirming the analysis. The growth of the VM curve is hard to see on this scale but is clear in Fig. 4. Also shown in Fig. 5 are results for the i.i.d. Rayleigh case where all users have unit link gain. As discussed in Sec. III-B and [6], for Rayleigh fading $\eta_{i} \rightarrow 1 / \alpha$. Since $\alpha=2$ in Fig. 5 we observe convergence to 0.5 . In comparison, it is clear that the uniform and 3GPP models continue to grow. Hence, the opposite behavior occurs with ray-based models compared to Rayleigh. The growth of the VM curve is hard to see on this scale but is clear in Fig. 4.

In Fig. 6, we evaluate the $\mathrm{CH}$ and FP results for a UPA with $K=2$ and an increasing number of antennas. We adopt the angular models from [17]. The azimuth central angle follows a wrapped Gaussian distribution $\left(\phi_{c} \sim \mathcal{N}\left(0^{\circ}, \sigma_{c}^{2}\right)\right)$ with a Laplacian offset distribution $\left(\Delta_{c, s} \sim \mathcal{L}\left(1 / \sigma_{s}\right)\right)$. Both the central and offset angles for elevation have Laplacian distributions with $\theta_{c} \sim \mathcal{L}\left(1 / \hat{\sigma}_{c}\right)$ (centred on $\left.90^{\circ}\right)$ and $\delta_{c, s} \sim \mathcal{L}\left(1 / \hat{\sigma}_{s}\right)$, respectively. We adopt the following parameters: the number of clusters is $C=20$, the number of subrays within a cluster is $L=20, \sigma_{c}=31.64^{\circ}$ and $\sigma_{s}=24.25^{\circ}$ from [30] and we 

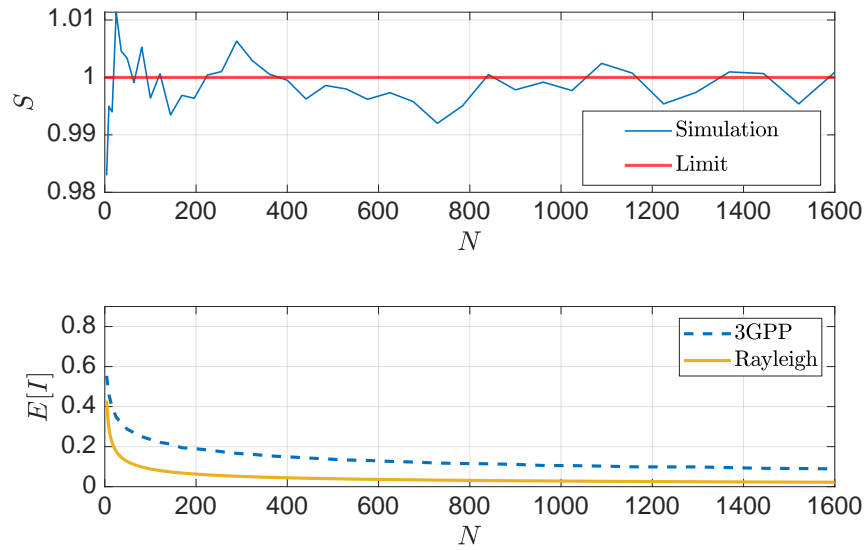

Fig. 6. Channel hardening and FP for UPA.

use the upper $90 \%$ of the lognormally distributed values of $\hat{\sigma}_{c}$ and $\hat{\sigma}_{s}$ from [17] in order to give a wide angular spread, which is $6.12^{\circ}$ and $1.84^{\circ}$, respectively (Scenario Wide). As in Fig. 1 and Fig. 2, equal subray powers are assumed and $\beta_{i r}=1 /(C L)$ for simplicity. From Fig. 6 , we see that, similar to ULA, FP and $\mathrm{CH}$ also occur for the UPA structure. However, the gap between the 3GPP and Rayleigh channel in Fig. 6 is wider than for a ULA. Hence, the smaller azimuth footprint of the UPA slows down FP.

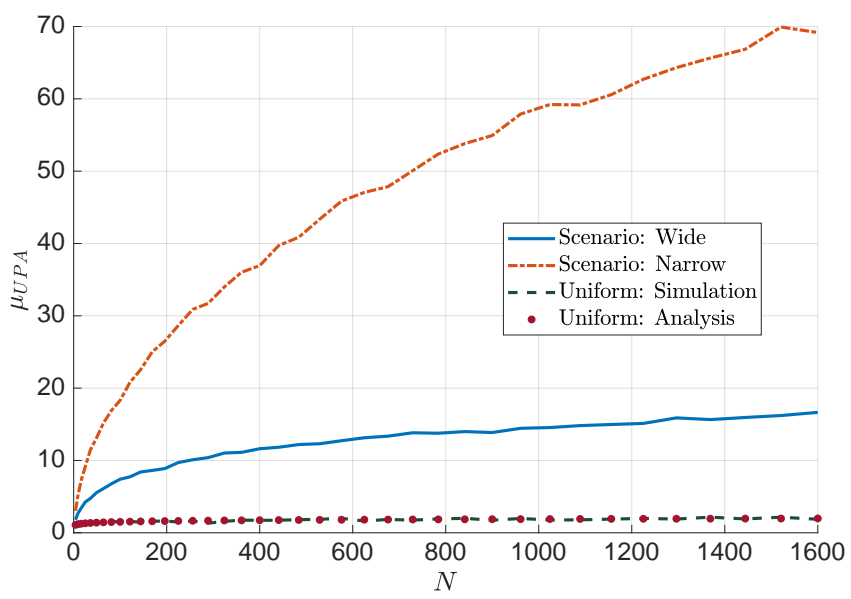

Fig. 7. $\mu_{\mathrm{UPA}}$ vs $N$ for wide and narrow angular distributions for UPA.

Fig. 7 shows the simulated and analytical results for $\mu_{\mathrm{UPA}}$ with a uniform angular distribution for both azimuth $(U[0,2 \pi])$ and elevation angles $(U[0, \pi])$. The analytical results are from Sec. IV-C. We see that the analysis agrees well with the simulation. We also show the simulated values of $\mu_{\mathrm{UPA}}$ for two scenarios. Scenario Wide uses the angular parameters in Fig. 6. Scenario Narrow uses $C=3, L=16, \sigma_{c}=14.4^{\circ}$ and $\sigma_{s}=6.24^{\circ}$ and the lower $10 \%$ of the lognormally distributed values of $\hat{\sigma}_{c}$ and $\hat{\sigma}_{s}$ from [17]. As with the ULA, the growth rates of $\mu_{\mathrm{UPA}}$ are different for all three models, due to the differences in azimuth and elevation angular distributions.

Fig. 8 shows the logarithmic growth of $\mu_{\mathrm{UPA}}$ with different angular spreads in both azimuth and elevation angles. As we can see, the narrower the angular spread, the quicker $\mu_{\text {UPA }}$

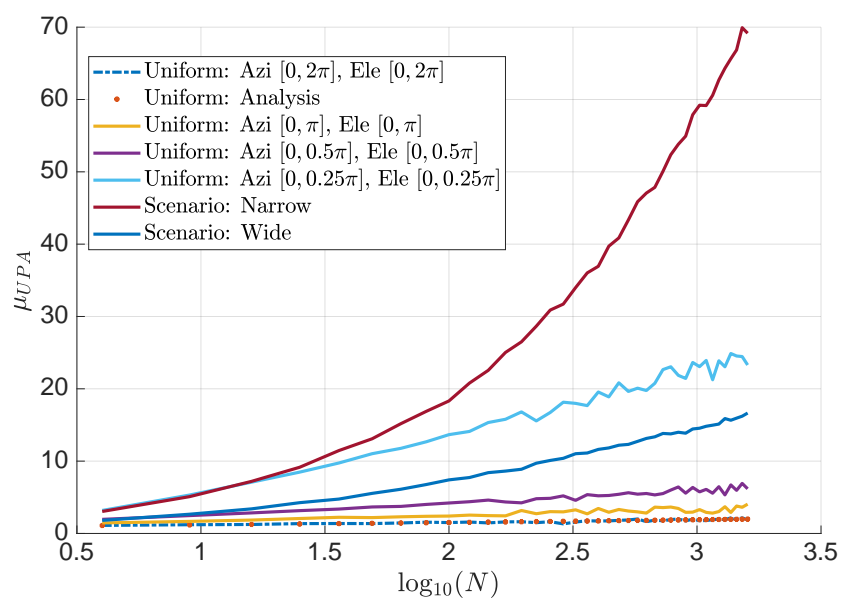

Fig. 8. Logarithmic growth of $\mu_{\mathrm{UPA}}$ for UPA.

grows. Note that the lower curves have stabilized and show linear growth with $\log _{10}(N)$ while the narrow scenario has not yet reached the high $N$ regime where logarithmic growth observed.

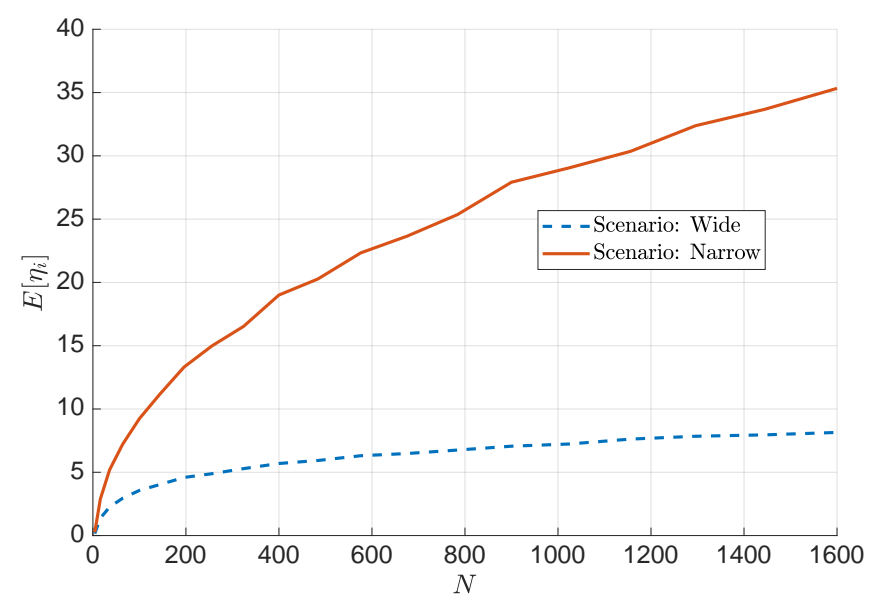

Fig. 9. $\mathbb{E}\left[\eta_{i}\right]$ vs $N$ for two angular distributions for UPA.

In Fig. 9, we confirm via simulation for the 3GPP parameters (adopting Scenario Wide and Scenario Narrow parameters of Fig. 7) that the mean global interference, $\mathbb{E}\left[\eta_{i}\right]$, grows logarithmically as predicted by the analysis in Sec. IV-C. We consider unequal ray powers and unequal user link gains, as in Fig. 5. Figs. 7- 9 show the logarithmic growth of the interference which is sufficient to show that $\zeta_{\text {LSP }} \rightarrow 0$ as $N \rightarrow \infty$. For completeness, in Fig. 10 we also plot $\zeta_{\text {LSP }}$ against $N$ for the same parameters used in Fig. 9 with the exception that both $\alpha=2$ and $\alpha=4$ are shown. On the $x$ axis, the array size, $N$, ranges from 16 (a $4 \times 4$ horizontal UPA) to 1600 (a $40 \times 40$ array). As expected, $\zeta_{\text {LSP }}$ decays more quickly for the more challenging scenarios, ie. smaller $\alpha$ and narrower angular spread. After the initial drop the decay to zero is slow as the interference growth is only logarithmic.

In Fig. 11, we show the instantaneous behavior of $\eta_{i}$ that causes $\mathbb{E}\left[\eta_{i}\right]$ to grow. A simple channel is assumed with 20 paths, half-wavelength antenna spacing in a ULA, unit power rays, $\alpha=10$ and all rays have a $U[0,2 \pi]$ distribution. With 


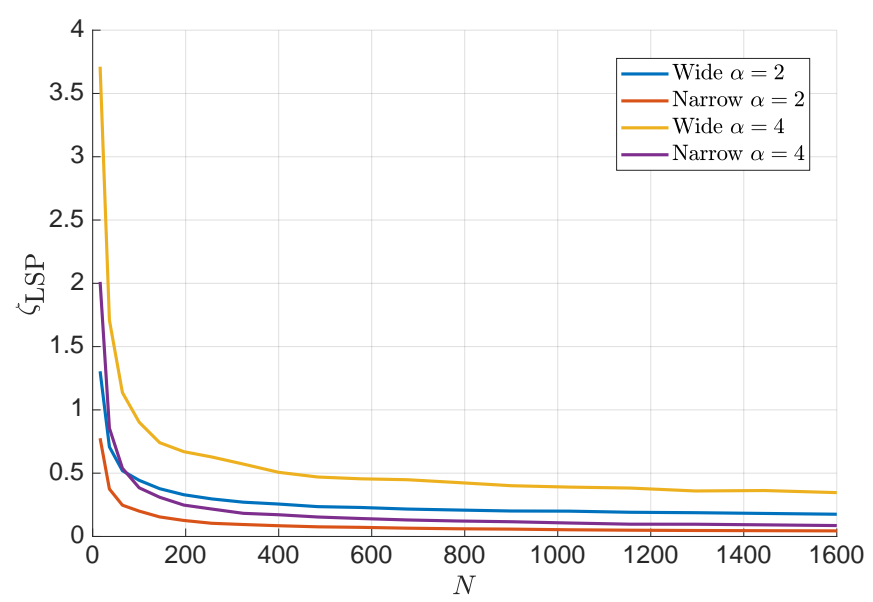

Fig. 10. $\zeta_{\text {LSP }}$ vs $N$ for two angular distributions for UPA.

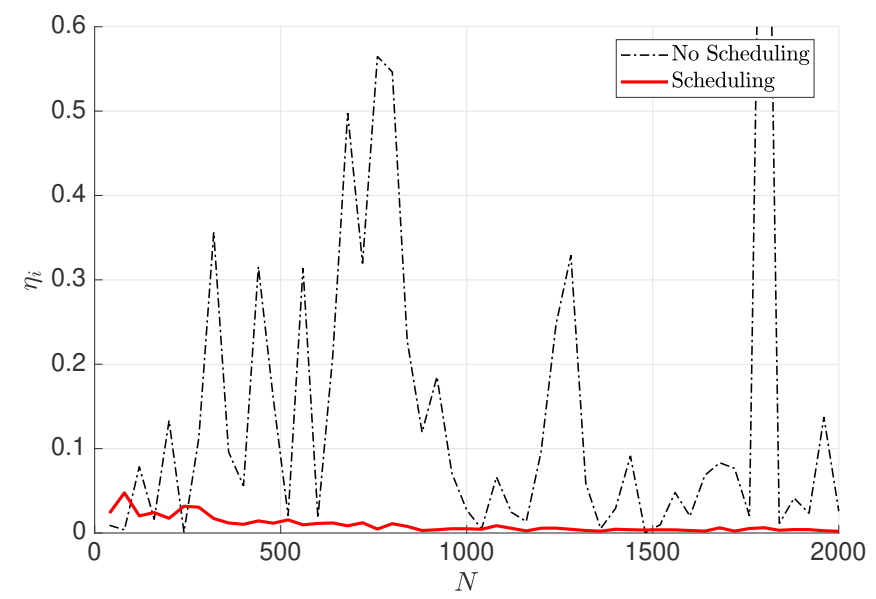

Fig. 11. $\eta_{i}$ vs $N$ with and without scheduling.

no control over the users entering the system, occasional large interference values occur as interfering user rays nearly align with the desired user. This is observed by the spikes in the curve labelled "no scheduling." Note that these spikes keep occurring even for massive antenna numbers, up to 2000. In contrast, we show the trivial scheduling scheme introduced in Sec. III-D with a protection target of $\epsilon=0.1$, equivalent to an angular separation of $0.57^{\circ}$. Here, the value of $\eta_{i}$ is wellbehaved and decays to zero.

\section{CONCLUSION}

The fundamental properties of massive MIMO have been identified with great generality for a broad class of ray-based models with a ULA or a UPA at the BS. The generality and insight possible is considerably greater than can be achieved with statistical channel models. In particular, we show that $\mathrm{CH}$ may or may not occur depending on the model used and FP is guaranteed for all continuous angular distributions. Although LSP will not normally hold, as the mean interference grows logarithmically relative to the desired channel, the implications for massive MIMO are excellent. As the number of users grows, the mean interference does grow relative to the desired channel but extremely slowly and this is further reduced by practical considerations, such as the attenuation of end-fire radiation caused by typical array patterns. In addition, we prove that this mean interference growth can be avoided by trivial scheduling schemes.

\section{APPENDIX A \\ PROOF OF THEOREM 1}

We note that

$$
\mathbb{E}\left[e^{-j q \hat{\phi}_{i r}}\right]=\int_{-2 \pi d}^{2 \pi d} e^{-j q x} f_{\hat{\phi}}(x) d x
$$

where $f_{\hat{\phi}}(\cdot)$ is PDF of $\hat{\phi}_{i r}$. Now, $\hat{\phi}_{i r}=2 \pi d \sin \phi_{i r}$ is a non one-to-one transformation of $\phi_{i r}$. Using standard transformation theory, we obtain

$$
f_{\hat{\phi}}(x)=\frac{p(x)}{\sqrt{l^{2}-x^{2}}},-l \leq x \leq l,
$$

where $l=2 \pi d$ and

$$
p(x)= \begin{cases}f_{\phi}\left(\sin ^{-1}\left(\frac{x}{l}\right)\right)+f_{\phi}\left(\pi-\sin ^{-1}\left(\frac{x}{l}\right)\right), & x \geq 0 \\ f_{\phi}\left(\sin ^{-1}\left(\frac{x}{l}\right)\right)+f_{\phi}\left(-\pi-\sin ^{-1}\left(\frac{x}{l}\right)\right), & x<0 .\end{cases}
$$

Hence, (28) is rewritten as

$$
\mathbb{E}\left[e^{-j q \hat{\phi}_{i r}}\right]=\int_{-l}^{l} e^{-j q x} \frac{p(x)}{\sqrt{l^{2}-x^{2}}} d x .
$$

Using the notation in [31, Eq.1, p. 15], the Fourier transform (FT) of a function $f(x)$ is

$$
g(y)=\int_{-\infty}^{\infty} f(x) e^{-j 2 \pi x y} d x
$$

If we set $y=\frac{q}{2 \pi}$, then

$$
g\left(\frac{q}{2 \pi}\right)=\int_{-\infty}^{\infty} f(x) e^{-j q x} d x .
$$

Using the Heaviside function, $H(x)$, we can write (31) as a FT in the same format as (33),

$\mathbb{E}\left[e^{-j q \hat{\phi}_{i r}}\right]=\int_{-\infty}^{\infty} e^{-j q x}(H(x+l)-H(x-l)) \frac{p(x)}{\sqrt{l^{2}-x^{2}}} d x$.

Hence, defining $f(x)=(H(x+l)-H(x-l)) \frac{p(x)}{\sqrt{l^{2}-x^{2}}}$, allows $\mathbb{E}\left[e^{-j q \hat{\phi}_{i r}}\right]$ to be computed as the FT of $f(x)$.

This formulation is particularly useful as we can now leverage known results on the asymptotics of FTs as $q \rightarrow \infty$ [31]. These results depend on the singularities of $f(x)$ so we first discuss the nature of these singularities. Clearly, $f(x)$ has singularities at $x= \pm l$ and at any singularities of $p(x)$. Note that the singularities at $x= \pm l$ are infinite discontinuities (indicating that the value of $f(x)$ will grow infinitely large as $x$ approaches $\pm l$ ). In contrast, the singularities of $p(x)$ are never infinite discontinuities for any proposed, practical angular distribution models. Models such as the wrapped Gaussian have no singularities inside $(-l, l)$ while the Laplacian has only a non-differentiable point at the peak. Hence, the singularities at $x= \pm l$ are the worst. The general principle presented in [31, 
p. 55] is that the 'worst' singularity ${ }^{6}$ of a function contributes the leading term to the asymptotic expression for its FT. Thus in our case, we only need to consider the two singularities at $x= \pm l$. Near $x_{1}=-l, f(x)$ behaves like $F_{1}(x)=$ $H(x+l) p(-l)(2 l(l+x))^{-1 / 2}$ and similarly near $x_{2}=l$, $f(x)$ behaves like $F_{2}(x)=(1-H(x-l)) p(l)(2 l(l-x))^{-1 / 2}$. Rewriting, we obtain

$$
\begin{gathered}
F_{1}(x)=\frac{H(x+l) p(-l)}{\sqrt{2 l}}|x+l|^{-\frac{1}{2}}, \\
F_{2}(x)=\frac{p(l)}{\sqrt{2 l}}|x-l|^{-\frac{1}{2}}-\frac{H(x-l) p(l)}{\sqrt{2 l}}|x-l|^{-\frac{1}{2}} .
\end{gathered}
$$

From [31, Theorem 19, p. 52], we know that if a generalised function, $f(x)$, has a finite number of singularities at $\left\{x=x_{1}, x_{2}, x_{3}, \ldots, x_{m}\right\}$, and for each of them $f(x)-F_{m}(x)$ has absolutely integrable $N^{\text {th }}$ order derivatives in an interval including $x_{m}$, where $F_{m}(x)$ is a linear combination of functions of type $\left|x-x_{m}\right|^{\beta},\left|x-x_{m}\right|^{\beta} \operatorname{sgn}\left(x-x_{m}\right)$, $\left|x-x_{m}\right|^{\beta} \log \left|x-x_{m}\right|,\left|x-x_{m}\right|^{\beta} \log \left|x-x_{m}\right| \operatorname{sgn}\left(x-x_{m}\right)$, and if $f^{(N)}(x)$ is well behaved at infinity, then $g(y)$, the FT of $f(x)$, satisfies $g(y)=\sum_{m=1}^{M} G_{m}(y)+o\left(|y|^{-N}\right)$, as $|y| \longrightarrow \infty$, where $G_{m}(y)$ is the FT of $F_{m}(x)$. Using this,

$$
g\left(\frac{q}{2 \pi}\right) \sim G_{1}\left(\frac{q}{2 \pi}\right)+G_{2}\left(\frac{q}{2 \pi}\right),
$$

where $G_{1}$ and $G_{2}$ are the FTs of $F_{1}(x)$ and $F_{2}(x)$ in (35) and (36) and $\sim$ denotes asymptotic equivalence defined in [26, p. 15]. From [31, Table 1, p. 43], the FTs required are

$$
\begin{aligned}
\mathcal{F}\left(|x-l|^{-\frac{1}{2}}\right) & =e^{-2 \pi j l y}|y|^{-\frac{1}{2}}, \\
\mathcal{F}\left(H(x+l)|x+l|^{-\frac{1}{2}}\right) & =e^{2 \pi j l y-\frac{1}{4} j \pi \operatorname{sgn}(y)}|2 y|^{-\frac{1}{2}}, \\
\mathcal{F}\left(H(x-l)|x-l|^{-\frac{1}{2}}\right) & =e^{-2 \pi j l y-\frac{1}{4} j \pi \operatorname{sgn}(y)}|2 y|^{-\frac{1}{2}} .
\end{aligned}
$$

Using (38), we obtain

$$
\begin{aligned}
& g\left(\frac{q}{2 \pi}\right) \sim G_{1}\left(\frac{q}{2 \pi}\right)+G_{2}\left(\frac{q}{2 \pi}\right), \\
& =\sqrt{\frac{\pi}{l q}}\left(\frac{p(-l)}{\sqrt{2}} e^{j\left(l q-\frac{\pi}{4}\right)}+p(l) e^{-j l q}-\frac{p(l)}{\sqrt{2}} \sqrt{\frac{\pi}{q}} e^{-j\left(l q+\frac{\pi}{4}\right)}\right) .
\end{aligned}
$$

Substituting $p(l)=2 f_{\phi}\left(\frac{\pi}{2}\right), p(-l)=2 f_{\phi}\left(\frac{-\pi}{2}\right)$ and $l=2 \pi d$ into (39), and after some simplification we obtain the result in Theorem 1.

\section{APPENDIX B \\ PROOF OF THEOREM 2}

We drop the subscripts to re-express $M_{v, w}$ as follows,

$$
M=\mathbb{E}_{\theta}\left[\mathbb{E}_{\phi}\left[e^{-j 2 \pi \nu \sin \theta \sin \tilde{\phi}}\right]\right],
$$

where $\tilde{\phi}=\phi-\Delta+\frac{\pi}{2}$. We get

$$
\begin{aligned}
M & =\int_{-\frac{\pi}{2}}^{0}\left\{\mathbb{E}_{\phi}\left[e^{-j 2 \pi \nu|\sin \theta| \sin \tilde{\tilde{\phi}}}\right]\right\} f(\theta) d \theta \\
& +\int_{0}^{\frac{\pi}{2}}\left\{\mathbb{E}_{\phi}\left[e^{-j 2 \pi \nu|\sin (\theta)| \sin \tilde{\phi}}\right]\right\} f(\theta) d \theta
\end{aligned}
$$

\footnotetext{
${ }^{6}$ The singularity $x=x_{m}$ of a function, $f(x)$, is worst if $f(x)$ is of order $\left|x-x_{m}\right|^{\beta}$ near $x_{m}$ and $\beta$ is the smallest value for all singularities [31, p. 55].
}

where $\tilde{\tilde{\phi}}=-\tilde{\phi}$. Setting $q=\nu, d=|\sin \theta|$, allows us to use Theorem 1 to give the asymptotic version of the expected values in (40). Hence,

$$
\begin{aligned}
& M \sim \int_{-\frac{\pi}{2}}^{0} \frac{f(\theta)}{\sqrt{\nu|\sin \theta|}}\left\{f_{\tilde{\tilde{\phi}}}\left(-\frac{\pi}{2}\right) e^{j\left(2 \pi \nu|\sin \theta|-\frac{\pi}{4}\right)}\right\} d \theta \\
& +\int_{-\frac{\pi}{2}}^{0} \frac{f(\theta)}{\sqrt{\nu|\sin \theta|}}\left\{f_{\tilde{\tilde{\phi}}}\left(\frac{\pi}{2}\right) e^{-j\left(2 \pi \nu|\sin \theta|-\frac{\pi}{4}\right)}\right\} d \theta \\
& +\int_{0}^{\frac{\pi}{2}} \frac{f(\theta)}{\sqrt{\nu|\sin \theta|}}\left\{f_{\tilde{\phi}}\left(-\frac{\pi}{2}\right) e^{j\left(2 \pi \nu|\sin \theta|-\frac{\pi}{4}\right)}\right\} d \theta \\
& +\int_{0}^{\frac{\pi}{2}} \frac{f(\theta)}{\sqrt{\nu|\sin \theta|}}\left\{f_{\tilde{\phi}}\left(\frac{\pi}{2}\right) e^{-j\left(2 \pi \nu|\sin \theta|-\frac{\pi}{4}\right)}\right\} d \theta .
\end{aligned}
$$

Substituting $f_{\tilde{\tilde{\phi}}}\left(-\frac{\pi}{2}\right)=f_{\phi}(\Delta), f_{\tilde{\tilde{\phi}}}\left(\frac{\pi}{2}\right)=f_{\phi}(\Delta-\pi)$, $f_{\tilde{\phi}}\left(-\frac{\pi}{2}\right)=f_{\phi}(\Delta-\pi)$, and $f_{\tilde{\phi}}\left(\frac{\pi}{2}\right)=f_{\phi}(\Delta)$ into (41), we have

$$
\begin{gathered}
M \sim \frac{f_{\phi}(\Delta) e^{-j \frac{\pi}{4}}}{\sqrt{\nu}} I_{1}+\frac{f_{\phi}(\Delta-\pi) e^{j \frac{\pi}{4}}}{\sqrt{\nu}} I_{2} \\
+\frac{f_{\phi}(\Delta-\pi) e^{-j \frac{\pi}{4}}}{\sqrt{\nu}} I_{3}+\frac{f_{\phi}(\Delta) e^{j \frac{\pi}{4}}}{\sqrt{\nu}} I_{4},
\end{gathered}
$$

where $I_{1}^{*}=I_{2}=\int_{-\frac{\pi}{2}}^{0} \frac{f(\theta) e^{j 2 \pi \nu \sin \theta}}{\sqrt{|\sin \theta|}} d \theta$ and $I_{3}^{*}=I_{4}=$ $\int_{0}^{\frac{\pi}{2}} \frac{f(\theta) e^{-j 2 \pi \nu \sin \theta}}{\sqrt{\sin \theta}} d \theta$. Hence, we only need to compute $I=$ $\int_{0}^{\frac{\pi}{2}} \frac{g(\theta) e^{-j 2 \pi \nu \sin \theta}}{\sqrt{\sin \theta}} d \theta$, where $g(\theta)=f(\theta)$ for $I_{3}$ and $I_{4}$ and $g(\theta)=f(-\theta)$ for $I_{1}$ and $I_{2}$. Let $X=\sin \theta$ and rewrite $I$ as

$$
I=\int_{0}^{1} \frac{g\left(\sin ^{-1}(x)\right) e^{-j 2 \pi \nu x}}{\sqrt{x} \sqrt{1-x^{2}}} d x=\int_{0}^{1} e^{-j 2 \pi \nu x} q(x) d x
$$

where $q(x)=\frac{g\left(\sin ^{-1}(x)\right)}{\sqrt{x} \sqrt{1-x^{2}}}[H(x)-H(x-1)]$. As in Theorem $1, q(x)$ has its worst singularities at $x \in\{0,1\}$. Using [31, p. 55] again, we have $q(x) \sim F_{1}(x)=g(0) H(x)|x|^{-\frac{1}{2}}$, when $x \longrightarrow 0$, and $q(x) \sim F_{2}(x)=-\frac{1}{\sqrt{2}} g\left(\frac{\pi}{2}\right)|x-1|^{-\frac{1}{2}}+$ $\frac{1}{\sqrt{2}} g\left(\frac{\pi}{2}\right)|x-1|^{-\frac{1}{2}} H(x-1)$, when $x \longrightarrow 1$. Thus, according to [31, Theorem 19, p. 52], we have $I \sim G_{1}(y)+G_{2}(y)$, where $G_{i}(\cdot)$ is the Fourier transform of $F_{i}(\cdot)$ and $y=\nu / 2 \pi$. Hence,

$$
\begin{aligned}
I & \sim g(0) \frac{e^{-j \frac{\pi}{4} \operatorname{sgn}(y)}}{\sqrt{2}}|y|^{-\frac{1}{2}}-g\left(\frac{\pi}{2}\right) \frac{e^{-j 2 \pi y}}{\sqrt{2}}|y|^{-\frac{1}{2}} \\
& +g\left(\frac{\pi}{2}\right) e^{-j 2 \pi y} \frac{e^{-j \frac{\pi}{4} \operatorname{sgn}(y)}}{2}|y|^{-\frac{1}{2}} .
\end{aligned}
$$

Substituting $\nu=2 \pi y$ in (44) and nothing that $\nu>0$ gives

$$
\begin{aligned}
I & \sim \frac{g(0) e^{-j \frac{\pi}{4}}}{\sqrt{2 \nu}}-\frac{g\left(\frac{\pi}{2}\right) e^{-j 2 \pi \nu}}{\sqrt{2 \nu}}+\frac{g\left(\frac{\pi}{2}\right) e^{-j 2 \pi \nu} e^{-j \frac{\pi}{4}}}{2 \sqrt{\nu}}, \\
& =\frac{1}{\sqrt{\nu}}\left\{g(0) e^{-j \frac{\pi}{4}}-\frac{g\left(\frac{\pi}{2}\right) e^{-j 2 \pi \nu+j \frac{\pi}{4}}}{2}\right\} .
\end{aligned}
$$


Substituting (45) into (42) we get

$$
\begin{aligned}
M & \sim \frac{f_{\phi}(\Delta) e^{-j \frac{\pi}{4}}}{\nu}\left\{\frac{f_{\theta}(0) e^{j \frac{\pi}{4}}}{\sqrt{2}}-\frac{f_{\theta}\left(-\frac{\pi}{2}\right)}{2} e^{j\left(2 \pi \nu-\frac{\pi}{4}\right)}\right\}, \\
& +\frac{f_{\phi}(\Delta-\pi) e^{j \frac{\pi}{4}}}{\nu}\left\{\frac{f_{\theta}(0) e^{-j \frac{\pi}{4}}}{\sqrt{2}}-\frac{f_{\theta}\left(-\frac{\pi}{2}\right) e^{-j\left(2 \pi \nu-\frac{\pi}{4}\right)}}{2}\right\}, \\
& +\frac{f_{\phi}(\Delta-\pi) e^{-j \frac{\pi}{4}}}{\nu}\left\{\frac{f_{\theta}(0) e^{j \frac{\pi}{4}}}{\sqrt{2}}-\frac{f_{\theta}\left(\frac{\pi}{2}\right) e^{j\left(2 \pi \nu-\frac{\pi}{4}\right)}}{2}\right\}, \\
& +\frac{f_{\phi}(\Delta) e^{j \frac{\pi}{4}}}{\nu}\left\{\frac{f_{\theta}(0) e^{-j \frac{\pi}{4}}}{\sqrt{2}}-\frac{f_{\theta}\left(\frac{\pi}{2}\right)}{2} e^{-j\left(2 \pi \nu-\frac{\pi}{4}\right)}\right\}, \\
& =\frac{j}{2 \nu}\left\{-f_{\phi}(\Delta-\pi) f_{\theta}\left(-\frac{\pi}{2}\right) e^{-j 2 \pi \nu}\right\}, \\
& +\frac{j}{2 \nu}\left\{f_{\phi}(\Delta-\pi) f_{\theta}\left(\frac{\pi}{2}\right) e^{j 2 \pi \nu}\right\}+\frac{j}{2 \nu}\left\{f_{\phi}(\Delta) f_{\theta}\left(-\frac{\pi}{2}\right) e^{j 2 \pi \nu}\right\}, \\
& -\frac{j}{2 \nu}\left\{f_{\phi}(\Delta) f_{\theta}\left(\frac{\pi}{2}\right) e^{-j 2 \pi \nu}\right\}+\frac{\sqrt{2} f_{\theta}(0)}{\nu}\left(f_{\phi}(\Delta)+f_{\phi}(\Delta-\pi)\right), \\
& \triangleq \frac{\alpha(\nu, \Delta)}{\nu} .
\end{aligned}
$$

According to a two-dimensional version of the integral test (a one-dimensional version of the integral test can be found in [32, Theorem 9.10, p. 619]), (22) converges if and only if the following expression converges as $N_{x} \rightarrow \infty, N_{y} \rightarrow \infty$,

$$
Z_{0}=\int_{-N_{x}}^{N_{x}} \int_{y \in \mathcal{Y}}\left(1-\frac{|x|}{N_{x}}\right)\left(1-\frac{|y|}{N_{y}}\right)\left|M_{x, y}\right|^{2} d y d x
$$

where $\mathcal{Y}=\left(-N_{y},-1\right) \cup\left(1, N_{y}\right)$. Note that the interval $[-1,+1]$ has been cut out of the integration zone for $y$. This is valid as it simply reflects that the first few terms indexed by $w$ in (22) are finite. As $M_{x, y} \sim \frac{\alpha(\nu, \Delta)}{\nu}$, we have

$$
Z_{0} \sim \int_{-N_{x}}^{N_{x}} \int_{y \in \mathcal{Y}}\left(1-\frac{|x|}{N_{x}}\right)\left(1-\frac{|y|}{N_{y}}\right) \frac{|\alpha(\nu, \Delta)|^{2}}{x^{2} d_{x}^{2}+y^{2} d_{y}^{2}} d y d x
$$

Since $|\alpha(\nu, \Delta)|^{2}$ is bounded and non-vanishing, the limit of $Z_{0}$ exists if and only if the limit of $Z_{1}$ exists, where

$$
\begin{aligned}
Z_{1} & \sim \int_{-N_{x}}^{N_{x}} \int_{y \in \mathcal{Y}}\left(1-\frac{|x|}{N_{x}}\right)\left(1-\frac{|y|}{N_{y}}\right) \frac{1}{x^{2}+y^{2}} d y d x \\
& =4 \int_{0}^{N_{x}} \int_{1}^{N_{y}}\left(1-\frac{x}{N_{x}}\right)\left(1-\frac{y}{N_{y}}\right) \frac{1}{x^{2}+y^{2}} d y d x \\
& =I_{A}+I_{B}+I_{C}+I_{D}
\end{aligned}
$$

where $I_{A}=\int_{0}^{N_{x}} \int_{1}^{N_{y}} \frac{1}{x^{2}+y^{2}} d y d x, \quad I_{B}=$ $-\int_{0}^{N_{x}} \int_{1}^{N_{y}} \frac{1}{N_{x}} \frac{x}{x^{2}+y^{2}} d y d x$

$I_{C}=\int_{0}^{N_{x}} \int_{1}^{N_{y}} \frac{1}{N_{x} N_{y}} \frac{x y}{x^{2}+y^{2}} d y d x$, and $I_{D}=$ $-\int_{0}^{N_{x}} \int_{1}^{N_{y}} \frac{1}{N_{y}} \frac{y}{x^{2}+y^{2}} d y d x$. Using the integral result $\int_{0}^{\infty}\left(x^{2}+y^{2}\right)^{-1} d x=\pi / 2 y$ [33, Eq. 2.124.1, p. 71], we are able to investigate the limits of $I_{A}, I_{B}, I_{C}$ and $I_{D}$. First, we consider $I_{D}$ :

$$
\begin{aligned}
\lim _{N_{x} \rightarrow \infty, N_{y} \rightarrow \infty} I_{D} & =-\lim _{N_{y} \rightarrow \infty} \int_{1}^{N_{y}} \frac{y}{N_{y}} \int_{0}^{\infty} \frac{y}{x^{2}+y^{2}} d x d y, \\
& =-\lim _{N_{y} \rightarrow \infty} \int_{1}^{N_{y}} \frac{\pi}{2 N_{y}} d y=-\frac{\pi}{2},
\end{aligned}
$$

which is finite. Similarly, $I_{B}$ and $I_{C}$ are also finite in the limit. Thus, the limiting behavior of (22) depends on $I_{A}$, and we have

$$
\begin{aligned}
\lim _{N_{x} \rightarrow \infty, N_{y} \rightarrow \infty} I_{A} & =\lim _{N_{y} \rightarrow \infty} \int_{1}^{N_{y}} \int_{0}^{\infty} \frac{1}{x^{2}+y^{2}} d x d y, \\
& =\lim _{N_{y} \rightarrow \infty} \int_{1}^{N_{y}} \frac{\pi}{2 y} d y=\lim _{N_{y} \rightarrow \infty} \frac{\pi}{2} \log \left(N_{y}\right) .
\end{aligned}
$$

Hence, $I_{A}$ grows logarithmically and (22) grows logarithmically as desired.

\section{APPENDIX C \\ DERIVATION OF $\mu_{\text {UPA }}$ FOR UNIFORM ANGULAR DISTRIBUTIONS}

In this scenario, beginning with the basic definition of $\mu_{\mathrm{UPA}}$, we have

$$
\begin{aligned}
\mu_{\mathrm{UPA}} & =\frac{1}{N} \mathbb{E}\left[\left|\mathbf{a}_{i r}^{\mathrm{H}} \mathbf{a}_{j s}\right|^{2}\right]=\frac{1}{N} \sum_{k=1}^{N} \sum_{l=1}^{N} \mathbb{E}\left[a_{i r k}^{*} a_{j s k} a_{j s l}^{*} a_{i r l}\right], \\
& =\frac{1}{N} \sum_{k=1}^{N} \sum_{l=1}^{N} \mathbb{E}\left[a_{i r k}^{*} a_{i r l}\right] \mathbb{E}\left[a_{j s l}^{*} a_{j s k}\right], \\
& =\frac{1}{N} \sum_{k=1}^{N} \sum_{l=1}^{N}\left|\mathbb{E}\left[a_{i r k}^{*} a_{i r l}\right]\right|^{2}, \\
& =\frac{1}{N} \sum_{k_{x}=1}^{N_{x}} \sum_{k_{y}=1}^{N_{y}} \sum_{l_{x}=1}^{l_{x}} \sum_{l_{y}=1}^{N_{y}}\left|\mathbb{E}\left[a_{i r x k_{x}}^{*} a_{i r y k_{y}}^{*} a_{i r x l_{x}} a_{i r y l_{y}}\right]\right|^{2} .
\end{aligned}
$$

The expectation in (50) is given by

$$
I_{1}=\mathbb{E}\left[\exp \left\{j 2 \pi \sin \theta\left(d_{y}\left(k_{y}-l_{y}\right) \sin \phi+d_{x}\left(k_{x}-l_{x}\right) \cos \phi\right)\right\}\right],
$$

Taking expectation over $\phi$ first gives an integral of the form

$$
\begin{aligned}
& \frac{1}{2 \pi} \int_{0}^{2 \pi} \exp \{j z(\alpha \sin \phi+\beta \cos \phi)\} d \phi \\
& =\frac{1}{2 \pi} \int_{0}^{2 \pi} \exp \left\{j z\left(\sqrt{\alpha^{2}+\beta^{2}} \sin (\phi+\Delta)\right)\right\} d \phi, \\
& =J_{0}\left(z \sqrt{\alpha^{2}+\beta^{2}}\right),
\end{aligned}
$$

where $\Delta=\tan ^{-1}(\beta / \alpha)$. Hence,

$$
\begin{aligned}
& I_{1}=\mathbb{E}\left[J_{0}\left(2 \pi \sin \theta \sqrt{d_{y}^{2}\left(k_{y}-l_{y}\right)^{2}+d_{x}^{2}\left(k_{x}-l_{x}\right)^{2}}\right)\right], \\
& =\frac{1}{\pi} \int_{0}^{\pi} J_{0}(\chi \sin \theta) d \theta, \\
& \text { where } \chi=2 \pi \sqrt{d_{y}^{2}\left(k_{y}-l_{y}\right)^{2}+d_{x}^{2}\left(k_{x}-l_{x}\right)^{2}} \text {. }
\end{aligned}
$$


From [33, Eq.6, p. 724], we have

$$
\int_{0}^{\pi} J_{0}(2 z \sin x) \cos (2 n x) d x=\pi J_{n}^{2}(x)
$$

Thus

$$
\int_{0}^{\pi} J_{0}(2 z \sin x) d x=\pi J_{0}^{2}(z)
$$

and we can rewrite $I_{1}$ as

$$
I_{1}=J_{0}^{2}\left(\pi \sqrt{d_{y}^{2}\left(k_{y}-l_{y}\right)^{2}+d_{x}^{2}\left(k_{x}-l_{x}\right)^{2}}\right) .
$$

Then, substituting $I_{1}$ into (50) allows $\mu_{\mathrm{UPA}}$ to be written as in (23).

\section{APPENDIX D}

\section{LOGARITHMIC GROWTH OF $\mu_{\text {UPA }}$ FOR UNIFORM ANGULAR DISTRIBUTIONS}

A simple change of indices, $m=r_{y}-s_{y}, n=r_{x}-s_{x}$ in (23) gives

$$
\mu_{\mathrm{UPA}}=\sum_{m=1-N_{y}}^{N_{y}-1} \sum_{n=1-N_{x}}^{N_{x}-1}\left(1-\frac{|m|}{N_{y}}\right)\left(1-\frac{|n|}{N_{x}}\right) J_{0}^{4}(A(m, n)),
$$

where $A(m, n)=\pi \sqrt{m^{2} d_{y}^{2}+n^{2} d_{x}^{2}}$. The sum in (57) is dominated by

$$
\mu_{1}=\sum_{m=1-N_{y}}^{N_{y}-1} \sum_{n=1-N_{x}}^{N_{x}-1} J_{0}^{4}(A(m, n)),
$$

and it is easy to show that the remaining terms in (57) are finite as $N \rightarrow \infty$. Hence, the asymptotic behavior of $\mu_{U P A}$ is the same as for $\mu_{1}$. Similarly, the sum in (58) is dominated by

$$
\mu_{2}=4 \sum_{m=1}^{N_{y}-1} \sum_{n=1}^{N_{x}-1} J_{0}^{4}(A(m, n))
$$

using the fact that $A(m, n)$ is an even function of $m$ and $n$ and neglecting finite terms. Again, the asymptotic behavior of $\mu_{\mathrm{UPA}}$ is the same as for $\mu_{2}$. Using the asymptotic equivalence [34, Eq. 10.17.3], $J_{0}(z) \sim \sqrt{\frac{2}{\pi z}} \cos (z-\pi / 4)$, we see that $\mu_{\text {UPA }}$ behaves like

$$
\begin{aligned}
\mu_{3} & =16 \sum_{m=1}^{N_{y}-1} \sum_{n=1}^{N_{x}-1} \frac{\cos ^{4}(A(m, n)-\pi / 4)}{\pi^{2} A^{2}(m, n)}, \\
& =\frac{16}{\pi^{4}} \sum_{m=1}^{N_{y}-1} \sum_{n=1}^{N_{x}-1} \frac{\cos ^{4}(A(m, n)-\pi / 4)}{m^{2} d_{y}^{2}+n^{2} d_{x}^{2}} .
\end{aligned}
$$

Application of a two-dimensional version of the integral test (one-dimensional version of the integral test can be found in [32, Theorem 9.10, p. 619]) and some further analysis to handle the oscillations in the $\cos ^{4}(\cdot)$ function via upper and lower bounds shows that the asymptotic behavior of $\mu_{3}$ is the same as that of

$$
\begin{aligned}
\mu_{4} & =\int_{1}^{N_{y}-1} \int_{1}^{N_{x}-1} \frac{1}{x^{2} d_{x}^{2}+y^{2} d_{y}^{2}} d_{x} d_{y}, \\
& =\frac{1}{d_{x} d_{y}} \int_{d_{y}}^{\left(N_{y}-1\right) d_{y}} \int_{d_{x}}^{\left(N_{x}-1\right) d_{x}} \frac{1}{u^{2}+v^{2}} d u d v .
\end{aligned}
$$

Converting to polar coordinates, a simple upper bound on (61) is

$$
\mu_{4} \leqslant \int_{0}^{\pi / 2} \int_{\rho_{\min }}^{\rho_{\max }} \frac{1}{\rho^{2}} \rho d \rho d \theta=\frac{\pi}{2}\left(\log \left(\rho_{\max }\right)-\log \left(\rho_{\min }\right)\right),
$$

where $\rho_{\min }=\min \left(d_{x}, d_{y}\right)$ and $\rho_{\max }=\sqrt{2} \max \left(\left(N_{x}-\right.\right.$ 1) $\left.d_{x},\left(N_{y}-1\right) d_{y}\right)$. Hence, the upper limit on $\mu_{4}$ grows logarithmically with $N$ as $N^{1 / 2} \leqslant \max \left(N_{x}, N_{y}\right) \leqslant N$, so that $\frac{1}{2} \log N \leqslant \log \left(\max \left(N_{x}, N_{y}\right)\right) \leqslant \log N$. Similarly, when $N_{x} \rightarrow \infty, N_{y} \rightarrow \infty$ as $N \rightarrow \infty, \mu_{4}$ can be lower bounded by a logarithmic function of $N$ by integrating over a sector of an annulus contained inside the integration region of (61). Hence, $\mu_{\text {UPA }}$ grows logarithmically with $N$ as required.

\section{REFERENCES}

[1] S. Li, P. J. Smith, P. A. Dmochowski, H. Tataria, M. Matthaiou, and J. Yin, "Massive MIMO for ray-based channels," in Proc. IEEE ICC, May 2019.

[2] H. Q. Ngo, E. G. Larsson, and T. L. Marzetta, "Energy and spectral efficiency of very large multiuser MIMO systems," IEEE Trans. Commun., vol. 61, no. 4, pp. 1436-1449, Apr. 2013.

[3] E. G. Larsson, O. Edfors, F. Tufvesson, and T. L. Marzetta, "Massive MIMO for next generation wireless systems," IEEE Commun. Mag., vol. 52, no. 2, pp. 186-195, Feb. 2014.

[4] H. Q. Ngo and E. G. Larsson, "No downlink pilots are needed in TDD massive MIMO," IEEE Trans. Wireless Commun., vol. 16, no. 5, pp. 2921-2935, May 2017.

[5] L. Liang, W. Xu, and X. Dong, "Low-complexity hybrid precoding in massive multiuser MIMO systems," IEEE Wireless Commun. Lett. vol. 3, no. 6, pp. 653-656, Dec. 2014.

[6] F. Rusek, D. Persson, B. K. Lau, E. G. Larsson, T. L. Marzetta, O. Edfors, and F. Tufvesson, "Scaling up MIMO: Opportunities and challenges with very large arrays," IEEE Signal Process. Mag., vol. 30, no. 1, pp. 40-60, Jan. 2013.

[7] E. Björnson, E. G. Larsson, and T. L. Marzetta, "Massive MIMO: Ten myths and one critical question," IEEE Commun. Mag., vol. 54, no. 2, pp. 114-123, Feb. 2016.

[8] E. Björnson, E. De Carvalho, J. H. Sørensen, E. G. Larsson, and P. Popovski, "A random access protocol for pilot allocation in crowded massive MIMO systems," IEEE Trans. Wireless Commun., vol. 16, no. 4, pp. 2220-2234, Apr. 2017.

[9] R. Couillet and M. Debbah, Random Matrix Methods for Wireless Communications. Cambridge University Press, 2011.

[10] Q. Zhang, S. Jin, K. K. Wong, H. Zhu, and M. Matthaiou, "Power scaling of uplink massive MIMO systems with arbitrary-rank channel means," IEEE J. Sel. Topics Signal Process., vol. 8, no. 5, pp. 966-981, Oct. 2014.

[11] M. Matthaiou, P. J. Smith, H. Q. Ngo, and H. Tataria, "Does massive MIMO fail in Ricean channels?" IEEE Wireless Commun. Lett., vol. 8, no. 1, pp. 61-64, Feb 2019.

[12] H. Tataria, P.J. Smith, M. Matthaiou, and P. A. Dmochowski, "Uplink analysis of large MU-MIMO systems with space-constrained arrays in Ricean fading," in Proc. IEEE ICC, May 2017, pp. 1-7.

[13] J. Li and Y. Zhao, "Measurement-based asymptotic user orthogonality analysis and modelling for massive MIMO," IEEE Commun. Lett., vol. 21, no. 12, pp. 2762-2765, Dec. 2017.

[14] J. Hoydis, C. Hoek, T. Wild, and S. ten Brink, "Channel measurements for large antenna arrays," in Proc. IEEE ISWCS, Aug. 2012, pp. 811815.

[15] X. Gao, O. Edfors, F. Rusek, and F. Tufvesson, "Massive MIMO performance evaluation based on measured propagation data." IEEE Trans. Wireless Commun., vol. 14, no. 7, pp. 3899-3911, Jul. 2015. 
[16] C. T. Neil, M. Shafi, P. J. Smith, P. A. Dmochowski, and J. Zhang, "Impact of microwave and mmWave channel models on $5 \mathrm{G}$ systems performance," IEEE Trans. Antennas Propag., vol. 65, no. 12, pp. 65056520, Dec. 2017.

[17] S. Sangodoyin, V. Kristem, C. U. Bas, M. Käske, J. Lee, C. Schneider, G. Sommerkorn, C. J. Zhang, R. Thomä, and A. F. Molisch, "Cluster characterization of 3-D MIMO propagation channel in an urban macrocellular environment," IEEE Trans. Wireless Commun., vol. 17, no. 8, pp. 5076-5091, May 2018.

[18] M. R. Akdeniz, Y. Liu, M. K. Samimi, S. Sun, S. Rangan, T. S. Rappaport, and E. Erkip, "Millimeter wave channel modeling and cellular capacity evaluation,” IEEE J. Sel. Areas Commun., vol. 32, no. 6, pp. 1164-1179, Jun. 2014.

[19] A. A. Saleh and R. Valenzuela, "A statistical model for indoor multipath propagation," IEEE J. Sel. Areas Commun., vol. 5, no. 2, pp. 128-137, Feb. 1987.

[20] X. Wu, N. C. Beaulieu, and D. Liu, "On favorable propagation in massive MIMO systems and different antenna configurations," IEEE Access, vol. 5, pp. 5578-5593, 2017.

[21] Z. Gao, L. Dai, C. Yuen, and Z. Wang, "Asymptotic orthogonality analysis of time-domain sparse massive MIMO channels," IEEE Commun. Lett., vol. 19, no. 10, pp. 1826-1829, Oct. 2015.

[22] M. Roy, S. Paquelet, L. L. Magoarou, and M. Crussière, "MIMO channel hardening: A physical model based analysis," arXiv preprint arXiv:1804.07491, 2018.

[23] H. Q. Ngo, E. G. Larsson, and T. L. Marzetta, "Aspects of favorable propagation in massive MIMO,” in Proc. EUSIPCO, Sep. 2014, pp. 7680

[24] J. Zhang, L. Tian, R. Xu, Z. Zhang, and J. Zhang, "Favorable propagation with practical angle distributions for mmWave massive MIMO systems," in Proc. IEEE ICC, May 2019.

[25] S. Buzzi and C. D'Andrea, "Energy efficiency and asymptotic performance evaluation of beamforming structures in doubly massive MIMO mmWave systems," IEEE Trans. Green Commun. Netw, vol. 2, no. 2, pp. 385-396, Jan. 2018.

[26] M. Abramowitz and I. A. Stegun, Handbook of Mathematical Functions: with Formulas, Graphs, and Mathematical Tables. Courier Corporation, 1964, vol. 55.

[27] H. Tataria and F. Tufvesson, "Impact of spatial consistency on dynamic beamforming for millimeter-wave cellular systems," in 9th MC and Technical Meeting of COST IRACON Action, Jan. 2019, pp. 1-9.

[28] S. Wang, B. R. Jackson, and R. Inkol, "Performance characterization of AOA geolocation systems using the Von Mises distribution," in Proc. IEEE VTC, Sep. 2012, pp. 1-5.

[29] S. Gunnarsson, J. Flordelis, L. Van der Perre, and F. Tufvesson, "Channel hardening in massive MIMO-A measurement based analysis," in Proc. IEEE SPAWC, June 2018.

[30] 3GPP, "Study on channel model for frequencies from 0.5 to $100 \mathrm{GHz}$," 3rd Generation Partnership Project (3GPP), Tech. Rep. TR 38.901 (V14.0.0), Mar. 2017. [Online]. Available: http://www.3gpp.org/

[31] M. J. Lighthill, An Introduction to Fourier Analysis and Generalised Functions. Cambridge University Press, 1958.

[32] R. Larson and B. H. Edwards, Calculus. Cengage Learning, 2009.

[33] I. S. Gradshteyn and I. M. Ryzhik, Table of Integrals, Series, and Products. Academic Press, 2014.

[34] F. W. Oliver, NIST Handbook of Mathematical Functions. Cambridge University Press, 2010. 